Supporting Information for

\title{
Asymmetric Reduction of Oxime Ethers Promoted by Chiral Spiroborate Esters with an $\mathrm{O}_{3} \mathrm{BN}$ Framework
}

\author{
Yunbo Chu, Zixing Shan*, Dejun Liu and Nannan Sun
}

\author{
S1 Contents
}

S2 General

S3 Preparation of oximes

S4-6 Preparation of oxime ethers

S7-S23 IR and ${ }^{1}$ H NMR spectra of oximes and oxime ethers

S24-S29 IR, ${ }^{1} \mathrm{H}$ NMR and ${ }^{13} \mathrm{C}$ NMR spectra of chiral amines

S30-S45 The chromatograms of acetylated derivative of the amines by HPLC analysis with a chiral column 


\section{General.}

Melting points were uncorrected. Column chromatography was performed on silica gel (200-300 mesh). $300 \mathrm{MHz}{ }^{1} \mathrm{H} \mathrm{NMR}$ and $75 \mathrm{MHz}{ }^{13} \mathrm{C}$ NMR spectra were recorded in $\mathrm{CDCl}_{3}$ in $\mathrm{ppm}$, $J$ in Hz. Optical rotations were recorded on digital polarimeter, Enantiomeric excess of the (S)-1-aralkylamines was determined for their acetylated derivative by HPLC analysis with a chiral column, flow rate $1.0 \mathrm{~mL} / \mathrm{min}$; hexane/2-propanol =90/10; UV, $254 \mathrm{~nm}$; the racemic aralkylamines were prepared according to a similar procedure to the literature. ${ }^{1}$ All the reductions were carried out under an atmosphere of dry Ar. Tetrahydrofuran (THF) was freshly distilled from sodium benzophenone under Ar prior to use. 1-(4-methoxyphenyl)ethanone, 1-(4-methoxy phenyl)propan-1-one and 1-(4-methoxy phenyl)butan-1-one were prepared according to the standard method. 


\section{Preparation of the oximes:}

A representative procedure is as follows

1-(4-methoxyphenyl)ethanone oxime: To a suspension of hydroxylamine hydrochloride $(4.17 \mathrm{~g})$ and anhydrous $\mathrm{K}_{2} \mathrm{CO}_{3}(8.28 \mathrm{~g})$ in $150 \mathrm{~mL}$ absolute ethanol was added 1-(4-methoxyphenyl)ethanone $(7.50 \mathrm{~g})$. The reaction mixture was stirred for $24 \mathrm{~h}$ under reflux, filtered, and then evaporated to remove ethanol. The residue was dissolved in water and extracted with diethyl ether (3 $\times 40 \mathrm{~mL}$ ). The combined ether layers were washed with saturated brine and dried over anhydrous $\mathrm{Na}_{2} \mathrm{SO}_{4}, 7.01 \mathrm{~g}$ of 1-(4-methoxyphenyl)ethanone oxime was afforded as white solid after evaporation of the solvent and column chromatography on silica gel (petroleum ether/ethyl acetate, $10: 2, \mathrm{v} / \mathrm{v}), 85 \%$ yield, mp. $76-78^{\circ} \mathrm{C}$. IR $\left(\mathrm{KBr}, \mathrm{cm}^{-1}\right): \mathrm{v} 3244,3007,2965,2936,2839,1610,1574$, 1516, 1461, 1368, 1258, 1175, 1025, 922, 831. ${ }^{1} \mathrm{H}$ NMR $\left(\mathrm{CDCl}_{3}\right): \delta 2.27$ (s, 3H), 3.84 (s, 3H), 6.90 $(\mathrm{d}, J=9.0 \mathrm{~Hz}, 2 \mathrm{H}), 7.58(\mathrm{~d}, J=9.0 \mathrm{~Hz}, 2 \mathrm{H}), 7.80(\mathrm{~s}, 1 \mathrm{H})$. Anal. Calcd. for $\mathrm{C}_{9} \mathrm{H}_{11} \mathrm{NO}_{2}$ : C, 65.44; H, $6.71 ; \mathrm{N}, 8.48$. Found: C, 65.21; H, 6.69; N, 8.65.

Other oximes were prepared according to similar procedure.

1-(4-Methoxyphenyl)propan-1-one oxime: white solid, 87\% yield, mp 65-67 ${ }^{\circ} \mathrm{C}$. IR $\left(\mathrm{KBr}, \mathrm{cm}^{-1}\right)$ : v $3287,3065,2971,2938,2838,1607,1575,1515,1465,1299,1254,1180,1037,970,914,835 .{ }^{1} \mathrm{H}$ NMR ( $\left.\mathrm{CDCl}_{3}\right): \delta 1.06$ (t, J=6.9 Hz, 3H), 2.69(q, J=6.9 Hz, 2H), $3.73(\mathrm{~s}, 3 \mathrm{H}), 6.80(\mathrm{~d}, J=7.5 \mathrm{~Hz}, 2 \mathrm{H})$, 7.46 (d, J=7.5 Hz, 2H), 8.52 (s, 1H). Anal. Calcd. for $\mathrm{C}_{10} \mathrm{H}_{13} \mathrm{NO}_{2}$ : C, 67.02; H, 7.31; N, 7.82. Found: C, 66.78; H, 7.26; N, 7.78.

1-(4-Methoxyphenyl)butan-1-one oxime: white solid, $82 \%$ yield, mp 54-56 ${ }^{\circ} \mathrm{C}$. IR $\left(\mathrm{KBr}, \mathrm{cm}^{-1}\right)$ : v $3286,3251,3069,2927,2871,2838,1601,1517,1464,1327,1260,1177,1029,935,827 .{ }^{1} \mathrm{H}$ $\operatorname{NMR}\left(\mathrm{CDCl}_{3}\right): \delta 0.98(\mathrm{t}, J=7.2 \mathrm{~Hz}, 3 \mathrm{H}), 1.60(\mathrm{~m}, J=7.2 \mathrm{~Hz}, 2 \mathrm{H}), 2.77(\mathrm{t}, J=7.2 \mathrm{~Hz}, 2 \mathrm{H}), 3.83(\mathrm{~s}$, $3 \mathrm{H}), 6.90(\mathrm{~d}, J=8.1 \mathrm{~Hz}, 2 \mathrm{H}), 7.56(\mathrm{~d}, J=8.1 \mathrm{~Hz}, 2 \mathrm{H})$. Anal. Calcd. for $\mathrm{C}_{11} \mathrm{H}_{15} \mathrm{NO}_{2}: \mathrm{C}, 68.37 ; \mathrm{H}, 7.82$; N, 7.25. Found: C, 68.28; H, 7.75; N, 7.21. 
Acetophenone oxime: white solid, $92 \%$ yield, mp 54-55 ${ }^{\circ} \mathrm{C}\left(\right.$ lit. $\left.^{2} \mathrm{mp} 59{ }^{\circ} \mathrm{C}\right)$. IR $\left(\mathrm{KBr}, \mathrm{cm}^{-1}\right): \mathrm{v}$ 3250, 3112, 3067, 3035, 2932, 1648, 1495, 1450, 1371, 1302, 1080, 1007, 927, 762, 694. ${ }^{1} \mathrm{H}$ NMR $\left(\mathrm{CDCl}_{3}\right): \delta 2.29(\mathrm{~s}, 3 \mathrm{H})$, 7.37-7.64 (m, 5H), $7.44(\mathrm{~s}, 1 \mathrm{H})$. Anal. Calcd. for $\mathrm{C}_{8} \mathrm{H}_{9} \mathrm{NO}: \mathrm{C}, 71.09 ; \mathrm{H}$, $6.71 ; \mathrm{N}, 10.36$. Found: C, 70.82; H, 6.56; N, 10.45.

Propiophenone oxime: white solid, $90 \%$ yield, $\mathrm{mp} 51-52{ }^{\circ} \mathrm{C}$. IR $\left(\mathrm{KBr}, \mathrm{cm}^{-1}\right): v \quad 3296,3250$, 3059, 2975, 2938, 2878, 1629, 1576, 1498, 1464, 1297, 1036, 971, 915, 767, 694. ${ }^{1} \mathrm{H} \mathrm{NMR}\left(\mathrm{CDCl}_{3}\right)$ : $\delta 1.18(\mathrm{t}, J=7.2 \mathrm{~Hz}, 3 \mathrm{H}), 2.84(\mathrm{~d}, J=7.2 \mathrm{~Hz}, 2 \mathrm{H}), 7.38-7.61(\mathrm{~m}, 5 \mathrm{H})$. Anal. Calcd. for $\mathrm{C}_{9} \mathrm{H}_{11} \mathrm{NO}$ : C, 72.46; H, 7.43; N, 9.39, Found: C, 72.34; H, 7.38; N, 9.42.

1-(4-Nitrophenyl)ethanone oxime: light yellow solid, $88 \%$ yield, mp 170-171 ${ }^{\circ} \mathrm{C}$ (lit. ${ }^{3} \mathrm{mp}$ 172-174 $\left.{ }^{\circ} \mathrm{C}\right)$. IR (KBr, cm $\left.{ }^{-1}\right): v 3253,3112,3078,2926,1601$ 1513, 1340, 1112, 1010, 930, 854, 774, 753, 691. ${ }^{1} \mathrm{H}$ NMR $\left(\mathrm{CDCl}_{3}\right): \delta 2.33(\mathrm{~s}, 3 \mathrm{H}), 7.81$ (d, J=7.5 Hz, 2H), $8.24(\mathrm{~d}, J=7.5 \mathrm{~Hz}, 2 \mathrm{H})$. Anal. Calcd. for $\mathrm{C}_{8} \mathrm{H}_{8} \mathrm{~N}_{2} \mathrm{O}_{3}$ : C, 53.33; H, 4.48; N, 15.55. Found: C, 53.12; H, 4.42; N, 15.45.

\section{Preparation of the oxime ethers:}

A representative procedure is as follows:

1-(4-Methoxyphenyl)ethanone O-methyl oxime. To a suspension of $\mathrm{NaH}(1.15 \mathrm{~g})$ in $100 \mathrm{~mL}$ dry N,N-dimethylformamide (DMF) was added 1-(4-methoxyphenyl)ethanone oxime (6.60 g) in dry $\operatorname{DMF}(50 \mathrm{~mL})$ at $0{ }^{\circ} \mathrm{C}$ (ice-bath). The reaction mixture was stirred for $2 \mathrm{~h}$ at $0{ }^{\circ} \mathrm{C}$ and $\mathrm{MeI}(6.82 \mathrm{~g})$ was added. The ice-bath was removed and the reaction mixture stirred for an additional $4 \mathrm{~h}$. After removal of DMF under reduced pressure, the residue was dissolved in water and extracted with diethyl ether $(3 \times 40 \mathrm{~mL})$. The combined ether layers were washed with saturated brine and dried anhydrous $\mathrm{Na}_{2} \mathrm{SO}_{4}$. After evaporation of the solvent and column chromatography on silica gel (petroleum ether/ethyl acetate, $16: 2$, v/v), 5.73 g 1-(4-methoxyphenyl)ethanone O-methyl oxime was afforded, $80 \%$ yield, mp 52-53 ${ }^{\circ} \mathrm{C}$ (lit. $\left.{ }^{4} \mathrm{mp} \mathrm{50-52}{ }^{\circ} \mathrm{C}\right)$. IR $\left(\mathrm{KBr}, \mathrm{cm}^{-1}\right): v 3003,2962,2941,2903$, 2839, 2822, 1613, 1599, 1516, 1458, 1444, 1257, 1175, 1049, 1026, 892, 828. ${ }^{1} \mathrm{H}$ NMR $\left(\mathrm{CDCl}_{3}\right): \delta$ 2.21(s, 3H), 3.83(s, 3H), 3.98(s, 3H), $6.88(\mathrm{~d}, J=8.7 \mathrm{~Hz}, 2 \mathrm{H}), 7.59$ (d, J=8.7 Hz, 2H). Anal. Calcd. for $\mathrm{C}_{10} \mathrm{H}_{13} \mathrm{NO}_{2}$ : C, 67.02; H, 7.31; N, 7.82. Found: C, 66.88; H, 7.28; N, 7.74.

Other oxime ethers were prepared similarly. 
(4-Methoxyphenyl)-propan-1-one O-methyl oxime, colourless oil, $84 \%$ yield. IR (neat, $\mathrm{cm}^{-1}$ ): v 3045, 2968, 2937, 2896, 2837, 2816, 1610, 1568, 1513, 1464, 1338, 1306, 1253, 1179, 1051, 958, 878, 835. ${ }^{1} \mathrm{H}$ NMR $\left(\mathrm{CDCl}_{3}\right): \delta 1.13(\mathrm{t}, J=7.2 \mathrm{~Hz}, 3 \mathrm{H}), 2.72(\mathrm{q}, J=7.2 \mathrm{~Hz}, 2 \mathrm{H}), 3.83(\mathrm{~s}, 3 \mathrm{H}), 3.96(\mathrm{~s}$, 3H), $6.89(\mathrm{~d}, J=9.0 \mathrm{~Hz}, 2 \mathrm{H}), 7.57(\mathrm{~d}, J=9.0 \mathrm{~Hz}, 2 \mathrm{H})$. Anal. Calcd. for $\mathrm{C}_{11} \mathrm{H}_{15} \mathrm{NO}_{2}: \mathrm{C}, 68.37 ; \mathrm{H}, 7.82$; N, 7.25. Found: C, 68.18; H, 7.78; N, 7.20.

1-(4-Methoxyphenyl)butan-1-one O-methyl oxime, colourless oil, $80 \%$ yield. IR (neat, $\mathrm{cm}^{-1}$ ): v 3045, 2961, 2935, 2899, 2873, 2837, 2816, 1611, 1567, 1514, 1464, 1303, 1251, 1178, 1052, 907, 888, 834. ${ }^{1} \mathrm{H} \mathrm{NMR}\left(\mathrm{CDCl}_{3}\right): \delta 0.97(\mathrm{t}, J=7.2 \mathrm{~Hz}, 3 \mathrm{H}), 1.56(\mathrm{~m}, J=7.2 \mathrm{~Hz}, 2 \mathrm{H}), 2.70(\mathrm{~m}, J=7.2 \mathrm{~Hz}$, 2H), $3.82(\mathrm{~s}, 3 \mathrm{H}), 3.96(\mathrm{~s}, 3 \mathrm{H}), 6.89(\mathrm{~d}, J=9.0 \mathrm{~Hz}, 2 \mathrm{H}), 7.59$ (d, J=9.0 Hz, 2H). Anal. Calcd. for $\mathrm{C}_{12} \mathrm{H}_{17} \mathrm{NO}_{2}$ : C, 69.54; H, 8.27; N, 6.76. Found: C, 69.32; H, 8.24; N, 6.62.

Acetophenone O-methyl oxime, colourless oil, 90\% yield. IR (neat, $\mathrm{cm}^{-1}$ ): v 3057, 2933, 2854, 2817, 1611, 1496, 1463, 1444, 1369, 1052, 893, 760, 693. ${ }^{1} \mathrm{H} \mathrm{NMR}\left(\mathrm{CDCl}_{3}\right): \delta 2.24$ (s, 3H), 4.02 (s, 3H), 7.36-7.64 (m, 5H). Anal. Calcd. for $\mathrm{C}_{9} \mathrm{H}_{11} \mathrm{NO}$ : C, 72.46; H, 7.43; N, 9.39. Found: C, 72.30; H, $7.38 ; \mathrm{N}, 9.29$.

Acetophenone O-benzyl oxime, colourless oil, 88\% yield. IR (neat, $\mathrm{cm}^{-1}$ ): $v$ 3061, 3031, 2926, 2872, 1608, 1496, 1449, 1367, 1311, 1021, 929, 880, 759, 695. ${ }^{1} \mathrm{H}$ NMR $\left(\mathrm{CDCl}_{3}\right): \delta 2.27(\mathrm{~s}, 3 \mathrm{H})$, $5.24(\mathrm{~s}, 2 \mathrm{H}), 7.30-7.65(\mathrm{~m}, 10 \mathrm{H})$. Anal. Calcd. for $\mathrm{C}_{15} \mathrm{H}_{15} \mathrm{NO}$ : C, 79.97; H, 6.71; N, 6.22. Found: C, 79.78; H, 6.65; N, 6.12 .

Propiophenone O-methyl oxime, colourless oil, 89\%. IR (neat, $\mathrm{cm}^{-1}$ ): v 3059, 2973, 2938, 2899, $2879,2817,1690,1597,1496,1463,1342,1220,1051,957,879,768,694 .{ }^{1} \mathrm{H} \mathrm{NMR}\left(\mathrm{CDCl}_{3}\right): \delta$ $1.13(\mathrm{t}, J=7.2 \mathrm{~Hz}, 3 \mathrm{H}), 2.75$ (q, $J=7.2 \mathrm{~Hz}, 2 \mathrm{H}), 3.98$ (s, 3H), 7.34-7.64 (m, 5H). Anal. Calcd. for $\mathrm{C}_{10} \mathrm{H}_{13} \mathrm{NO}: \mathrm{C}, 73.59 ; \mathrm{H}, 8.03 ; \mathrm{N}, 8.58$. Found: C, 73.32; H, 7.93; N, 8.48.

Propiophenone O-benzyl oxime, colourless oil, 84\% yield. IR (neat, $\mathrm{cm}^{-1}$ ): v 3060, 3031, 2974, 2936, 2876, 1606, 1571, 1496, 1453, 1366, 1306, 1022, 959, 873, 768, 740, 695. ${ }^{1} \mathrm{H} \mathrm{NMR}\left(\mathrm{CDCl}_{3}\right)$ : $\delta 1.14(\mathrm{t}, J=7.5 \mathrm{~Hz}, 3 \mathrm{H}), 2.79$ (q, J=7.5 Hz, 2H), 5.23 (s, 2H), 7.28-7.64 (m, 10H). Anal. Calcd. for $\mathrm{C}_{16} \mathrm{H}_{17} \mathrm{NO}: \mathrm{C}, 80.30 ; \mathrm{H}, 7.16$; N, 5.85. Found: C, 80.08; H, 7.12, N, 5.68. 
Propiophenone O-n-butyl oxime, colourless oil, 82\% yield. IR (neat, $\mathrm{cm}^{-1}$ ): $v$ 3059, 2960, 2935 , 2874, 1497, 1464, 1376, 1071, 1031, 965, 916, 886, 768, 694. ${ }^{1} \mathrm{H}$ NMR $\left(\mathrm{CDCl}_{3}\right): \delta 0.96$ (t, J=7.2 Hz, $3 \mathrm{H}), 1.14(\mathrm{t}, J=7.5 \mathrm{~Hz}, 3 \mathrm{H}), 1.44(\mathrm{~m}, J=7.5 \mathrm{~Hz}, 2 \mathrm{H}), 1.71(\mathrm{~m}, J=7.5 \mathrm{~Hz}, 2 \mathrm{H}), 2.75(\mathrm{~m}, J=7.5 \mathrm{~Hz}, 2 \mathrm{H})$, 4.18 (t, J=6.6 Hz, 2H). Anal. Calcd. for $\mathrm{C}_{13} \mathrm{H}_{19} \mathrm{NO}$ : C, 76.06, H, 9.33, N, 6.82. Found: C, 75.84; H, $9.18 ; \mathrm{N}, 6.75$.

1-(4-Nitrophenyl)ethanone O-methyl oxime, light brown solid, $81 \%$ yield, mp 108-109 ${ }^{\circ} \mathrm{C}$ (lit. ${ }^{4}$ $\left.\mathrm{mp} 110-112^{\circ} \mathrm{C}\right)$. IR $\left(\mathrm{KBr}, \mathrm{cm}^{-1}\right): v$ 3109, 3055, 3007, 2975, 2941, 2823, 1616, 1599, 1585, 1513, 1346, 1052, 904, 854, 752, 690. ${ }^{1} \mathrm{H}$ NMR $\left(\mathrm{CDCl}_{3}\right): \delta 2.26$ (s, 3H), 4.05 (s, 3H), $7.82(\mathrm{~d}, J=8.7 \mathrm{~Hz}, 2 \mathrm{H})$, 8.22 (d, J=8.7 Hz, 2H). Anal. Calcd. for $\mathrm{C}_{9} \mathrm{H}_{10} \mathrm{~N}_{2} \mathrm{O}_{3}: \mathrm{C}, 55.67 ; \mathrm{H}, 5.19 ; \mathrm{N}, 14.43$. Found: C, 55.45; H, $5.16 ; \mathrm{N}, 14.28$.

1-(4-Nitrophenyl)ethanone O-benzyl oxime, light yellow solid, $78 \%$ yield, mp $79-80{ }^{\circ} \mathrm{C}$. IR $\left(\mathrm{KBr}, \mathrm{cm}^{-1}\right): v$ 3086, 3053, 3031, 2948, 2883, 1596, 1581, 1515, 1344, 1020, 942, 854, 753, 699, 687. ${ }^{1} \mathrm{H}$ NMR $\left(\mathrm{CDCl}_{3}\right): \delta 2.30(\mathrm{~s}, 3 \mathrm{H}), 5.28(\mathrm{~s}, 2 \mathrm{H}), 7.33-7.41(\mathrm{~m}, 5 \mathrm{H}), 7.82(\mathrm{~d}, J=9.0 \mathrm{~Hz}, 2 \mathrm{H}), 8.21(\mathrm{~d}$, $J=9.0 \mathrm{~Hz}, 2 \mathrm{H})$. Anal. Calcd. for $\mathrm{C}_{15} \mathrm{H}_{14} \mathrm{~N}_{2} \mathrm{O}_{3}$ : C, 66.66; H, 5.22; N, 10.36. Found: C, 66.42; H, 5.15; $\mathrm{N}, 10.22$.

1-(4-Nitrophenyl)ethanone O-n-butyl oxime, light brown oil, 75\% yield. IR (neat, $\mathrm{cm}^{-1}$ ): $v 3081$, 2960, 2934, 2873, 1958, 1584, 1519, 1461, 1346, 1112, 1066, 1031, 938, 906, 855, 775, 753, 693. ${ }^{1} \mathrm{H}$ NMR $\left(\mathrm{CDCl}_{3}\right): \delta 0.97(\mathrm{t}, J=7.2 \mathrm{~Hz}, 3 \mathrm{H}), 1.42(\mathrm{~m}, J=7.2 \mathrm{~Hz}, 2 \mathrm{H}), 1.73(\mathrm{~m}, J=7.2 \mathrm{~Hz}, 2 \mathrm{H}), 2.26(\mathrm{~s}$, $3 \mathrm{H}), 4.25(\mathrm{t}, \mathrm{J}=7.2 \mathrm{~Hz}, 2 \mathrm{H}), 7.82(\mathrm{~d}, J=9.0 \mathrm{~Hz}, 2 \mathrm{H}), 8.21(\mathrm{~d}, \mathrm{~J}=9.0 \mathrm{~Hz}, 2 \mathrm{H})$. Anal. Calcd. for $\mathrm{C}_{12} \mathrm{H}_{16} \mathrm{~N}_{2} \mathrm{O}_{3}: \mathrm{C}, 61.00 ; \mathrm{H}, 6.83 ; \mathrm{N}, 11.86$. Found: C, 60.82; H, 6.58; N, 11.72.

\section{References:}

1 Ingersoll, A. W. Organic Syntheses, 1943, Coll. Vol. 2, 503.

2 (a) Coustard, J. M.; Jacquesy, J. C.; Violeau, B. Tetrahedron Lett. 1992, 33, 8085. (b) Smith, J. H.; Kaiser, E. T. J. Org. Chem.1974, 39, 729.

3 Uno, T.; Gong, B.; Schultz, P. G. J. Am. Chem. Soc.1994, 116, 1145.

4 Johnson, J. E.; Silk, N. M.; Arfan, M. J. Org. Chem.1982, 47, 1958. 

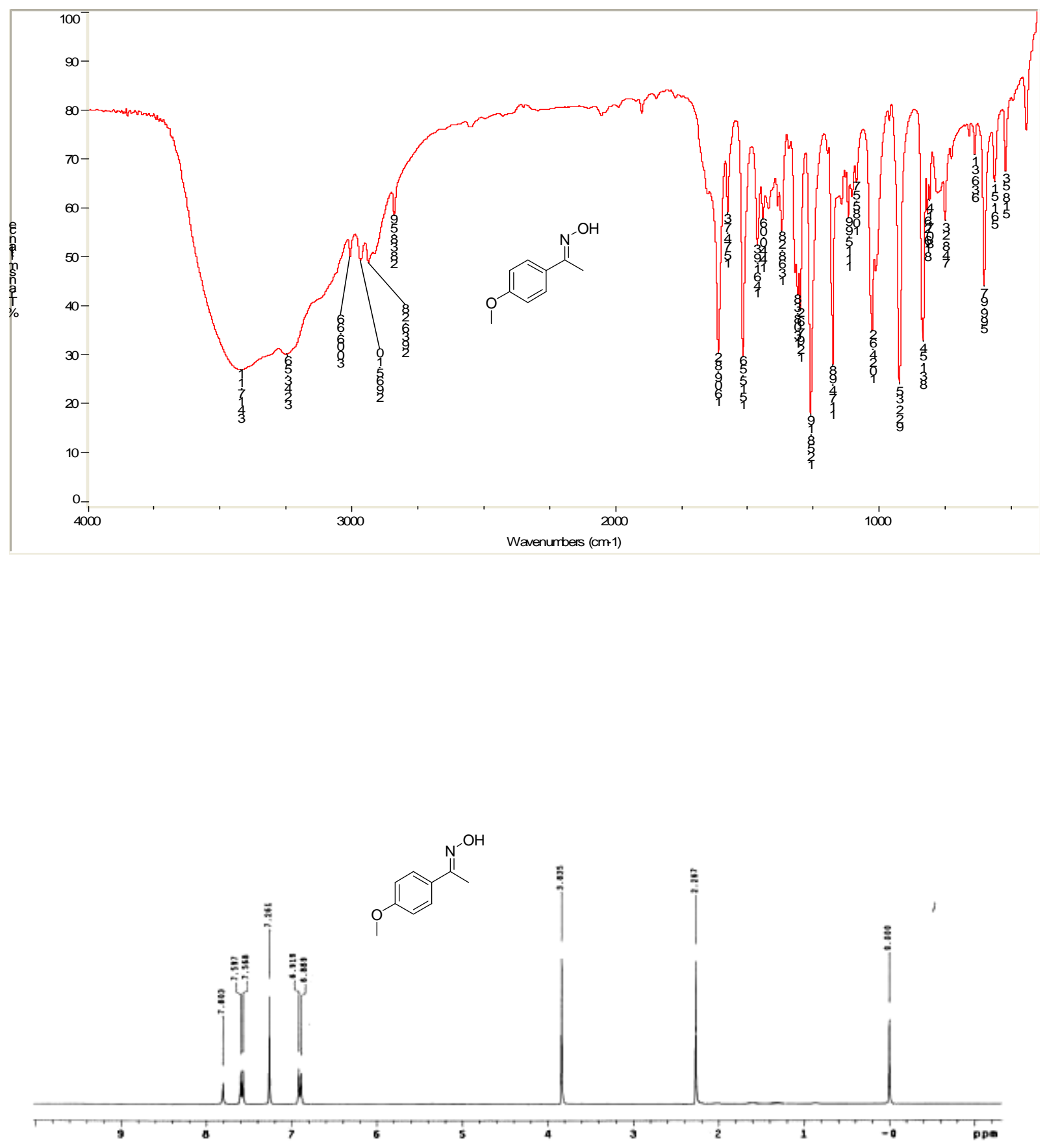

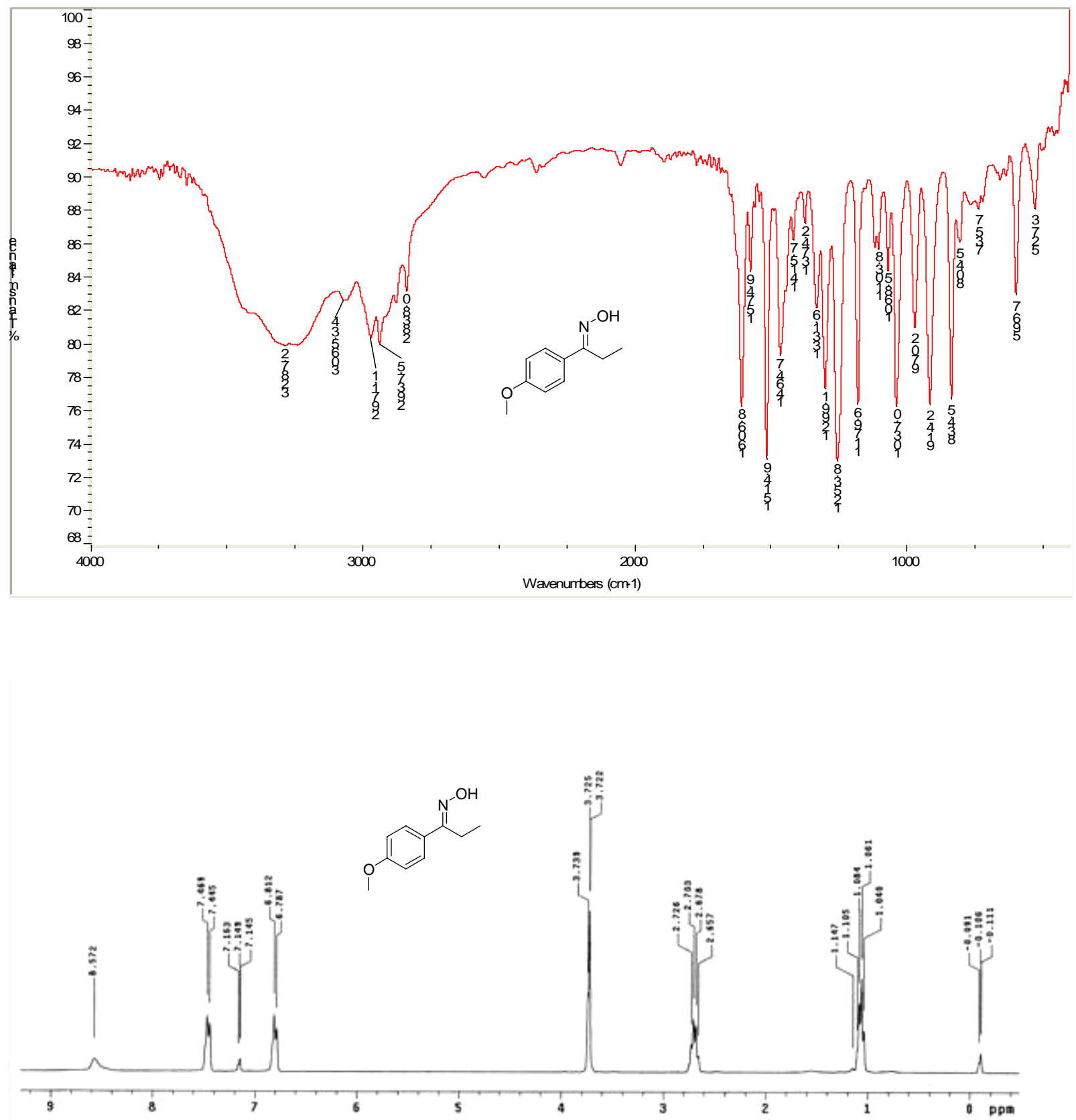

S8 

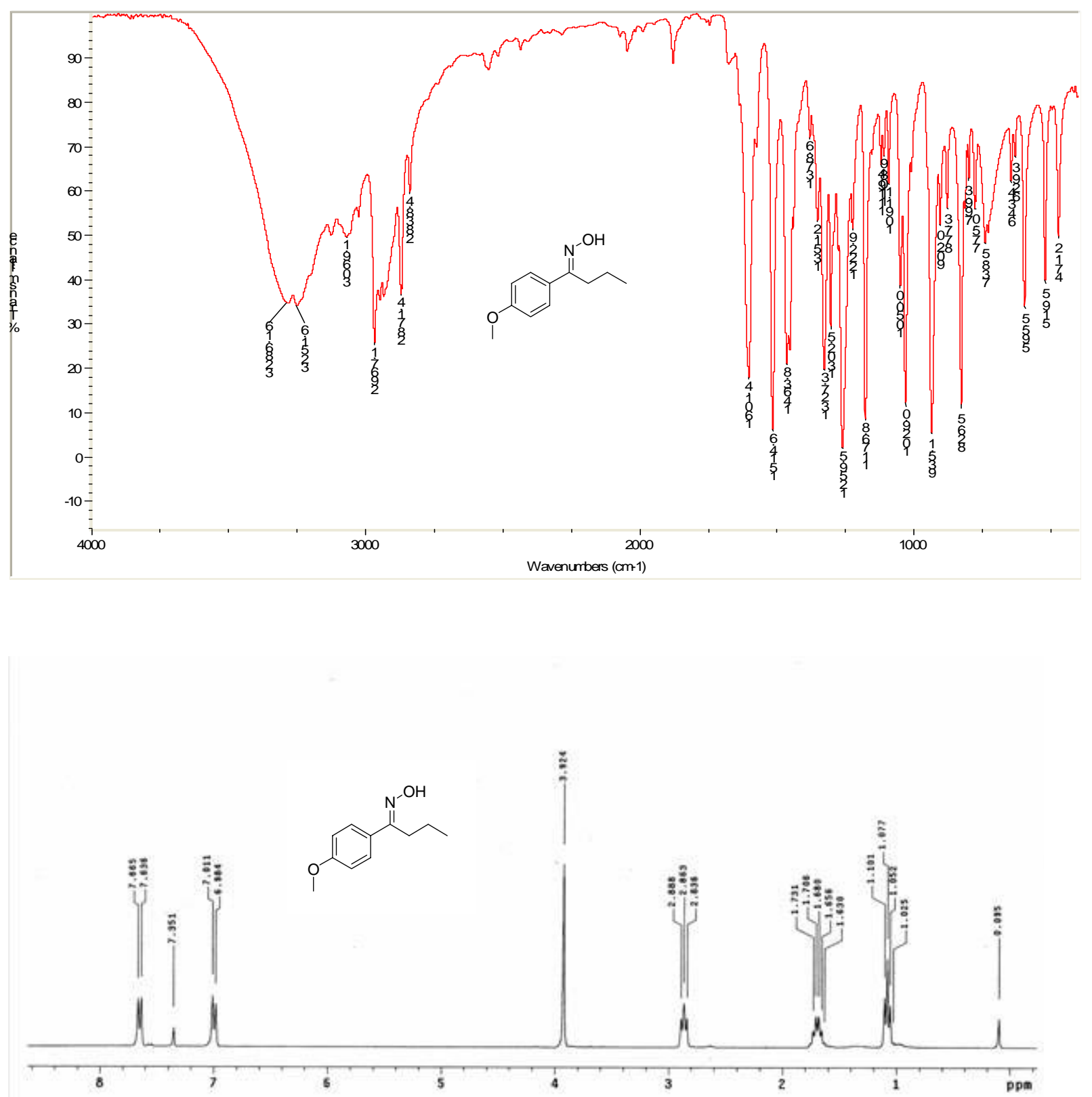

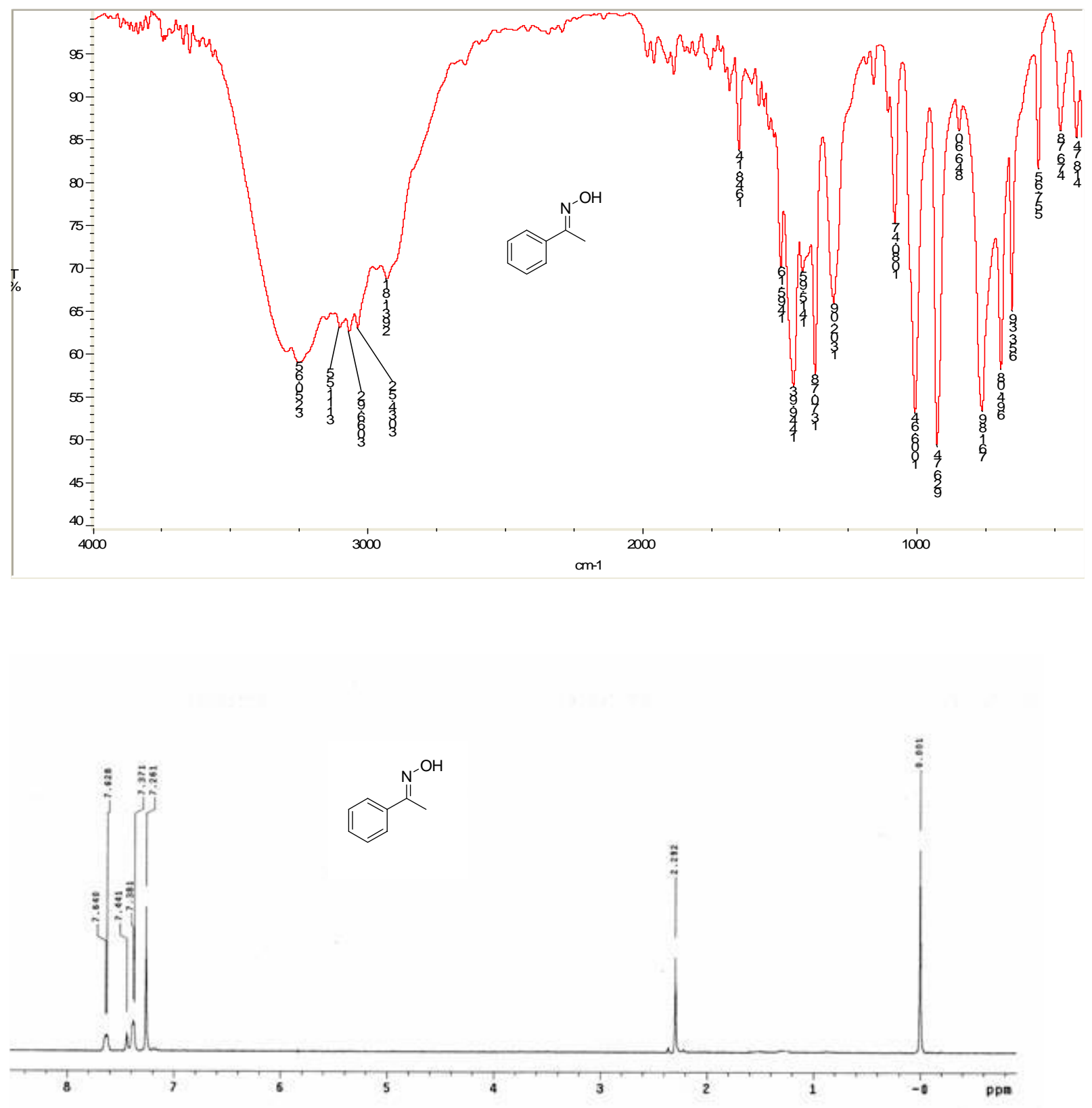

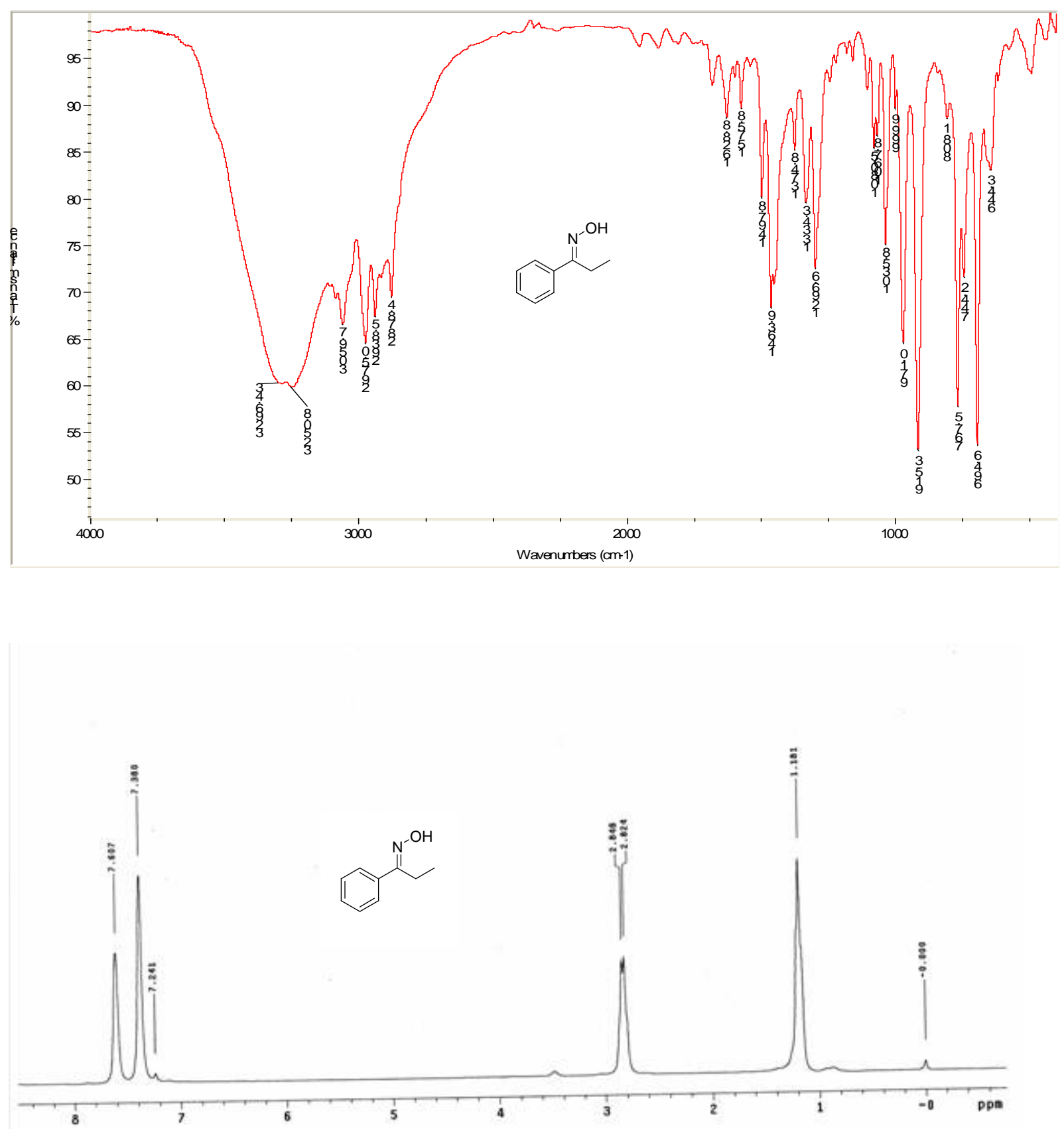

S11 

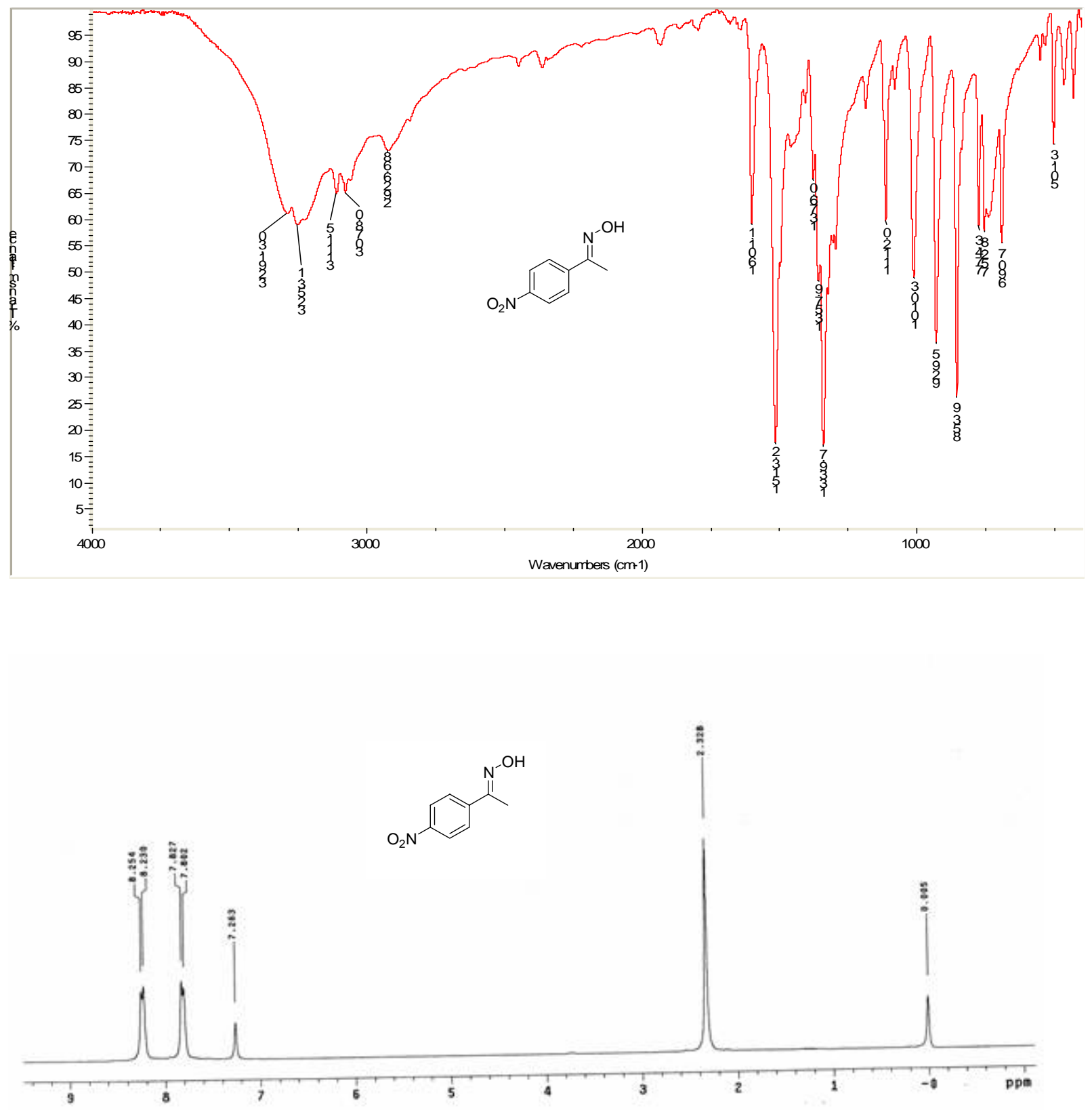

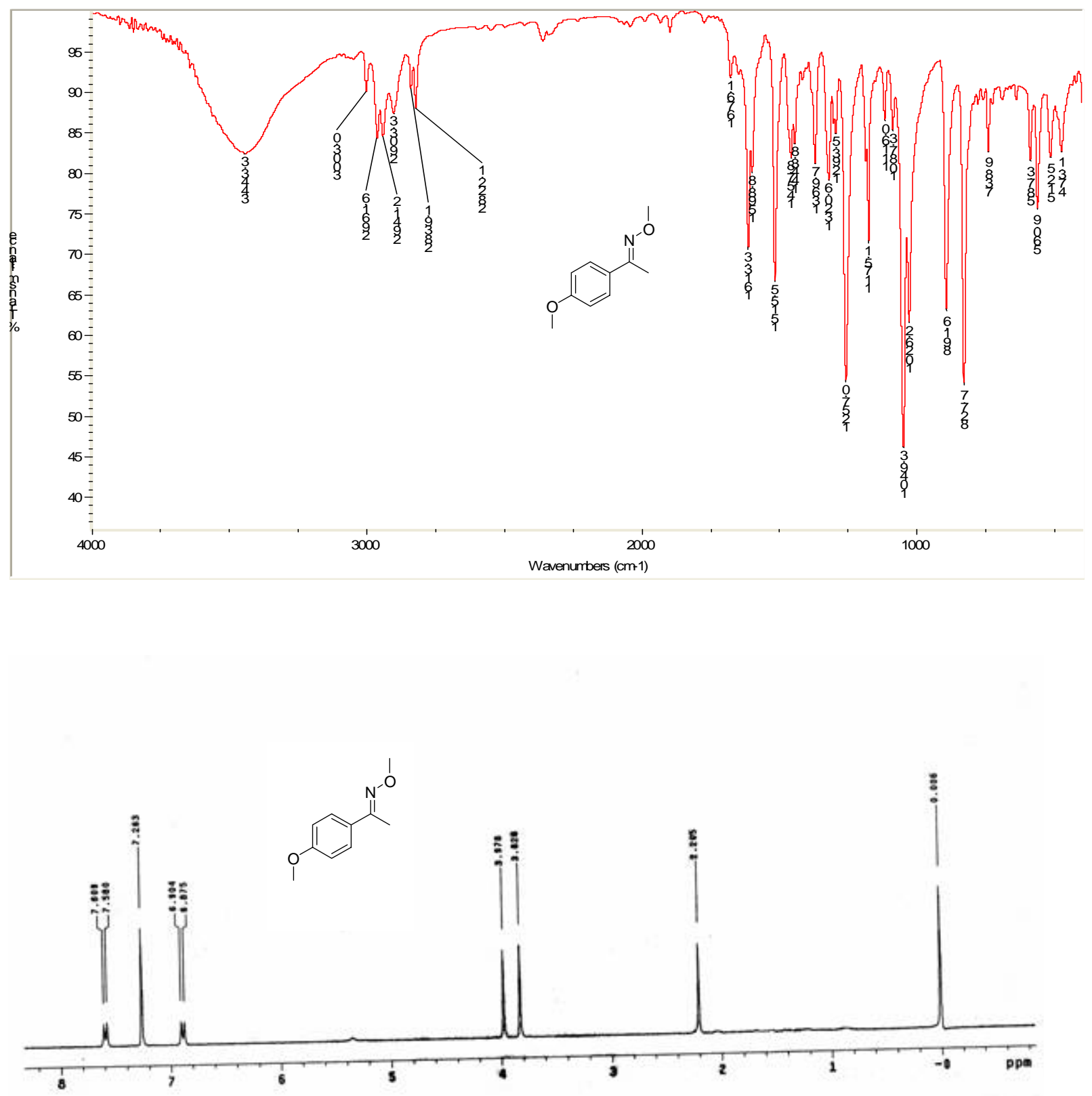

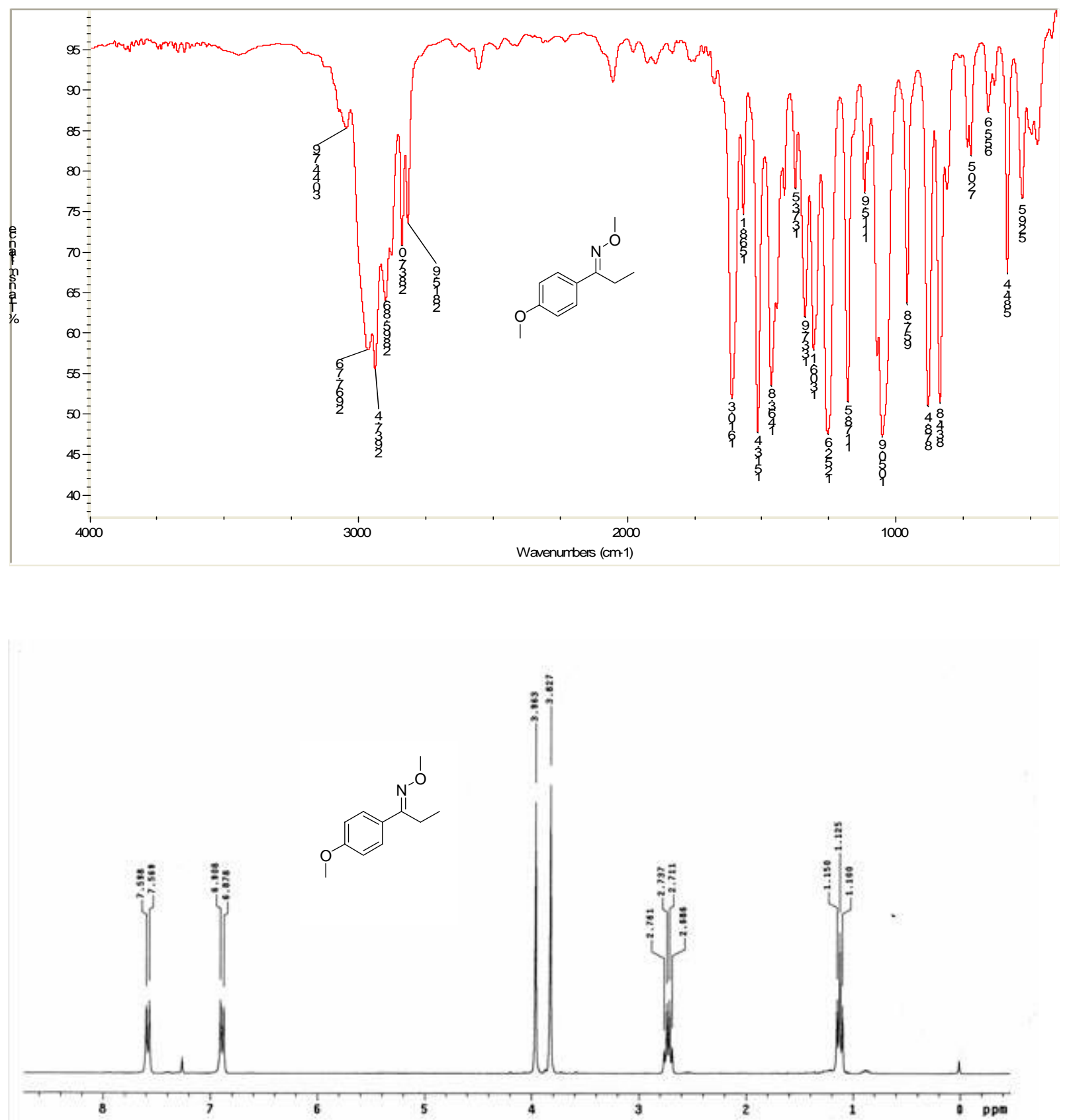

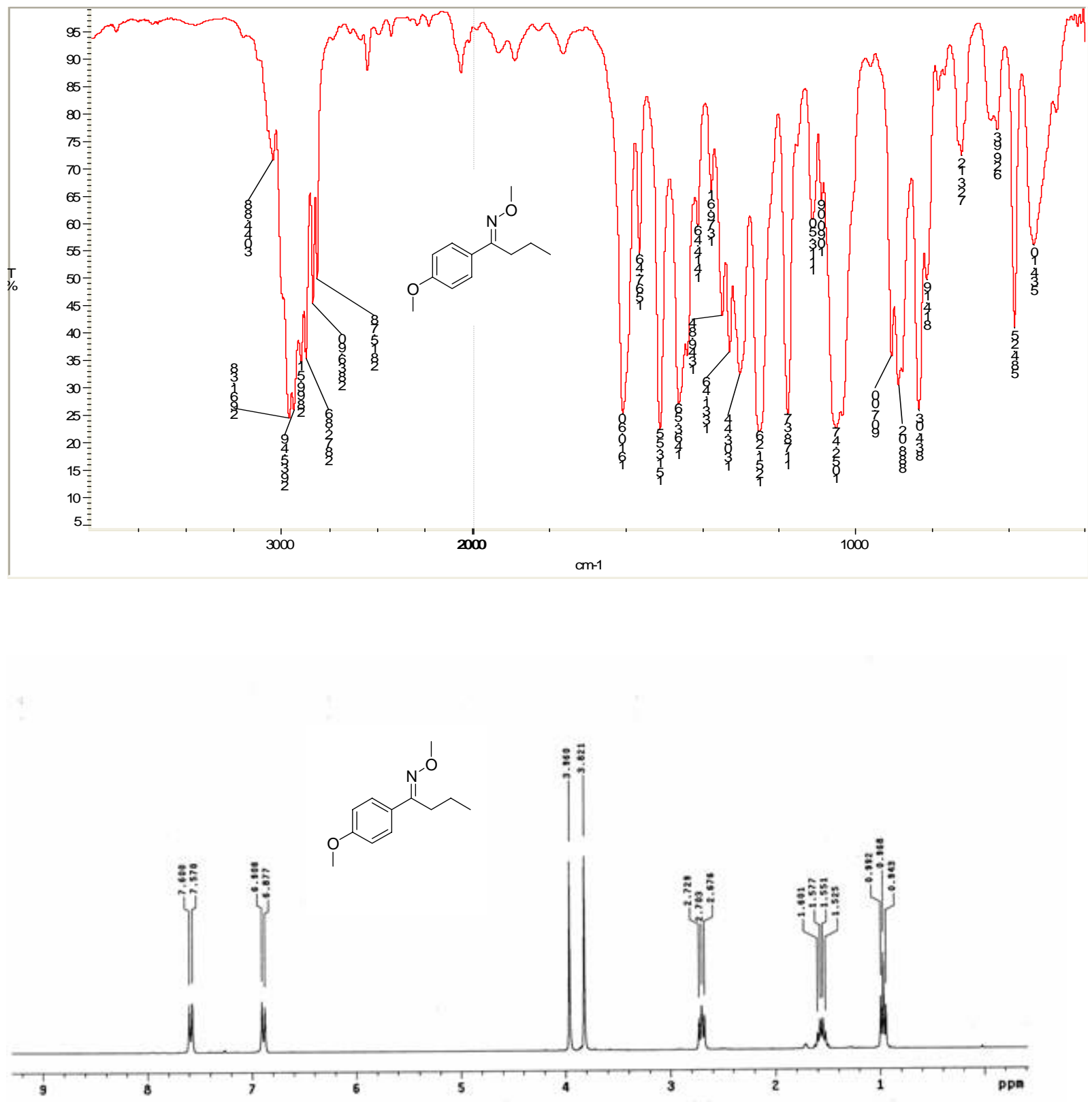

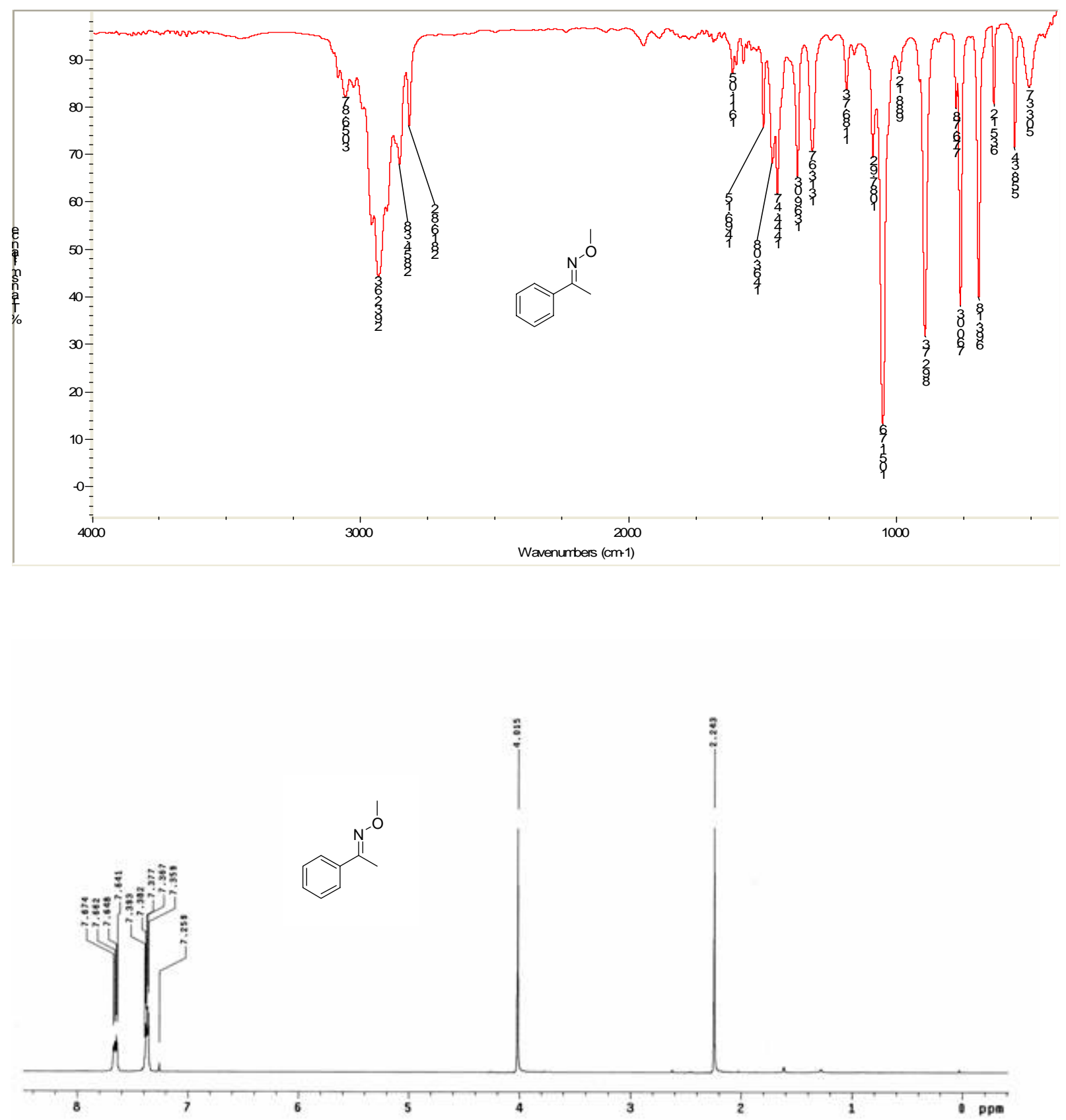

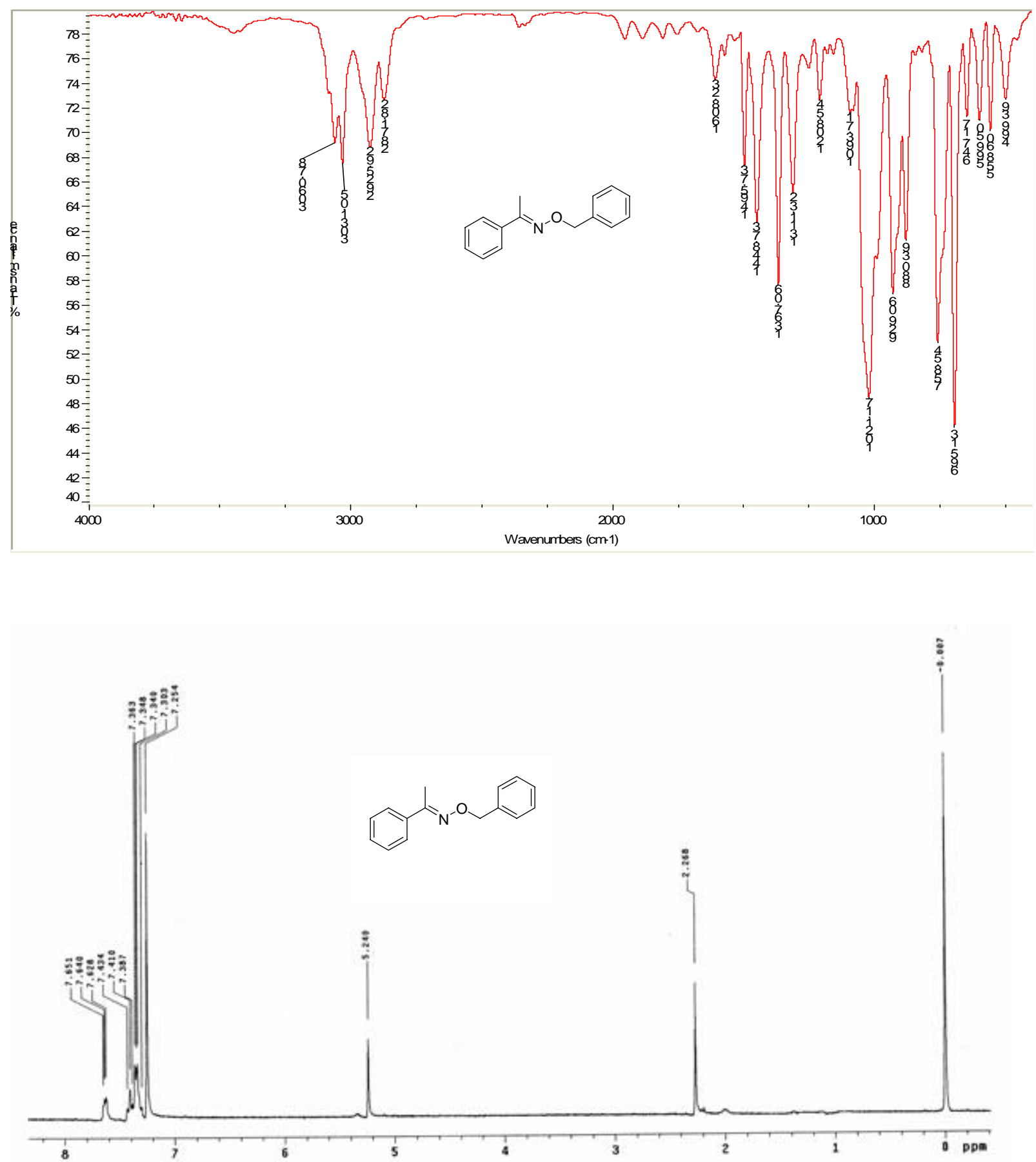

S17 

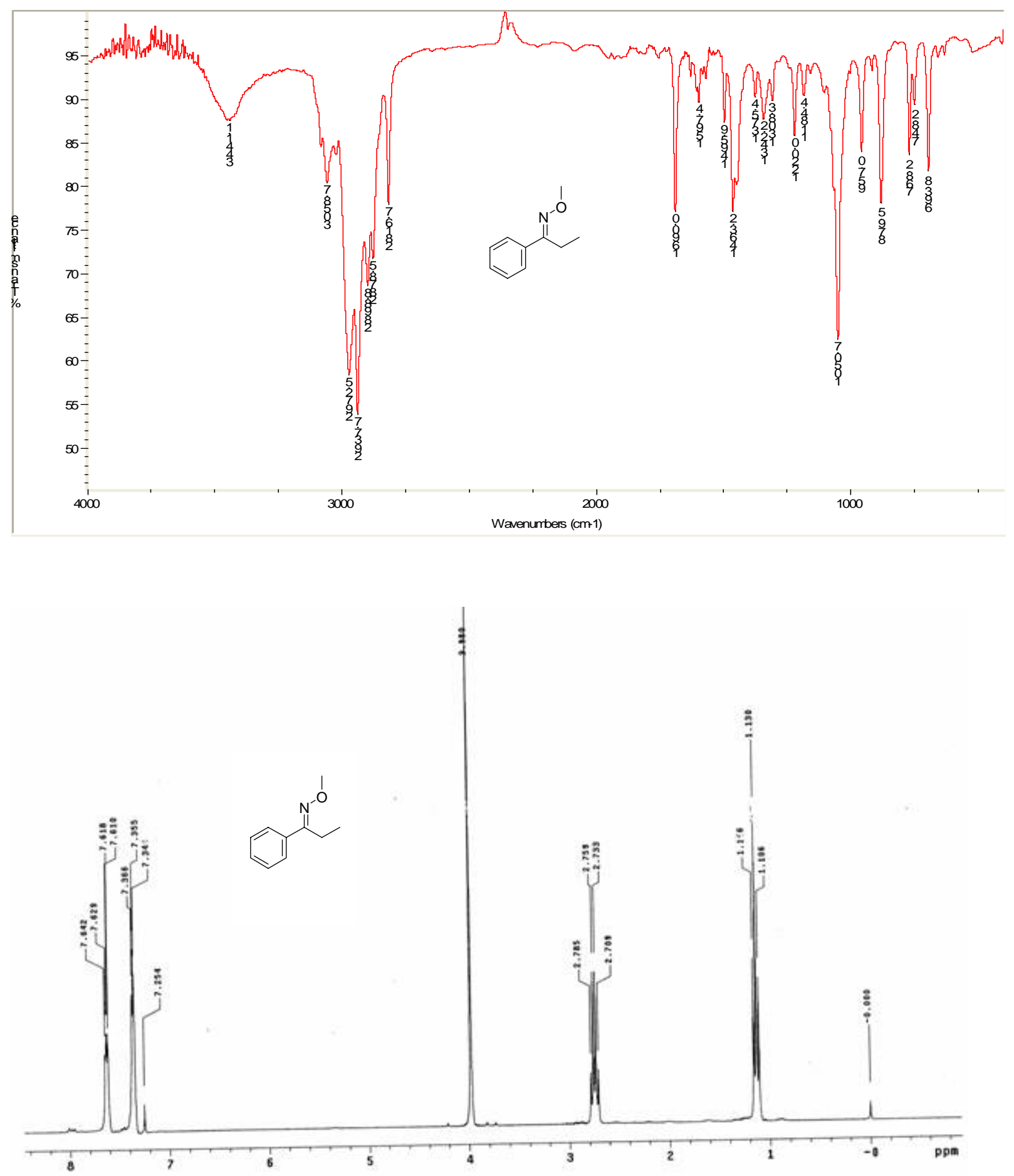

S18 

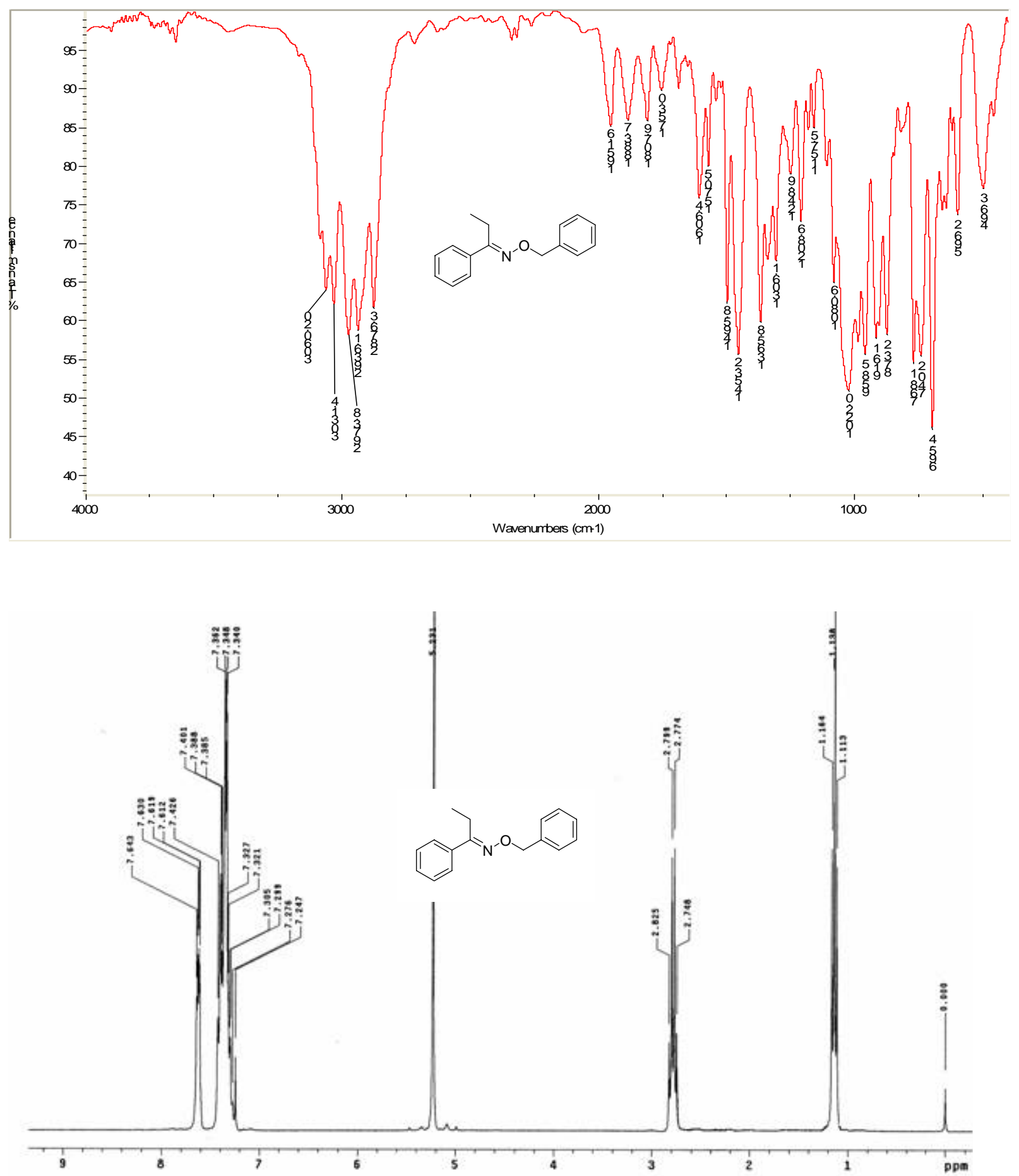

S19 

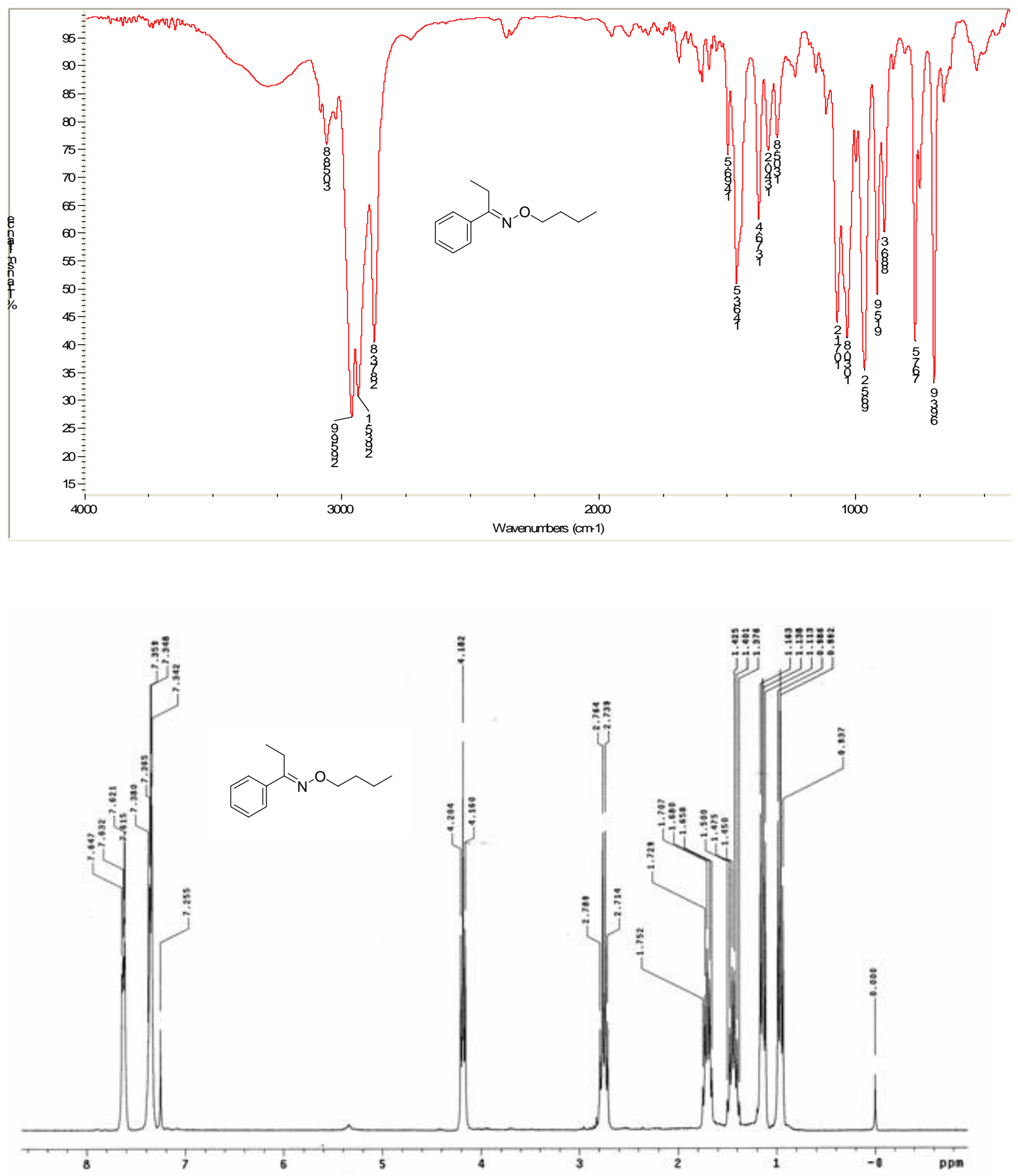

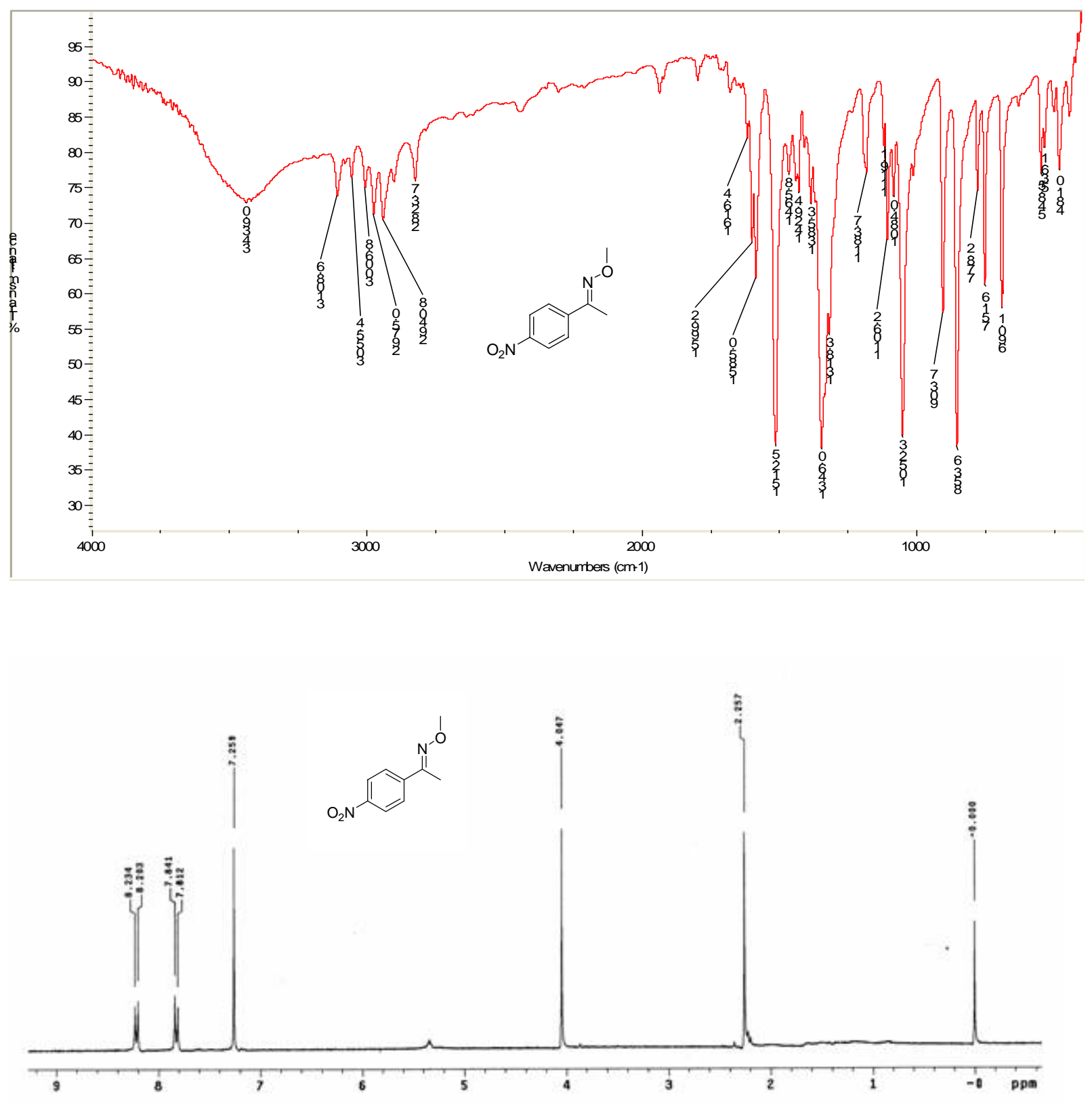

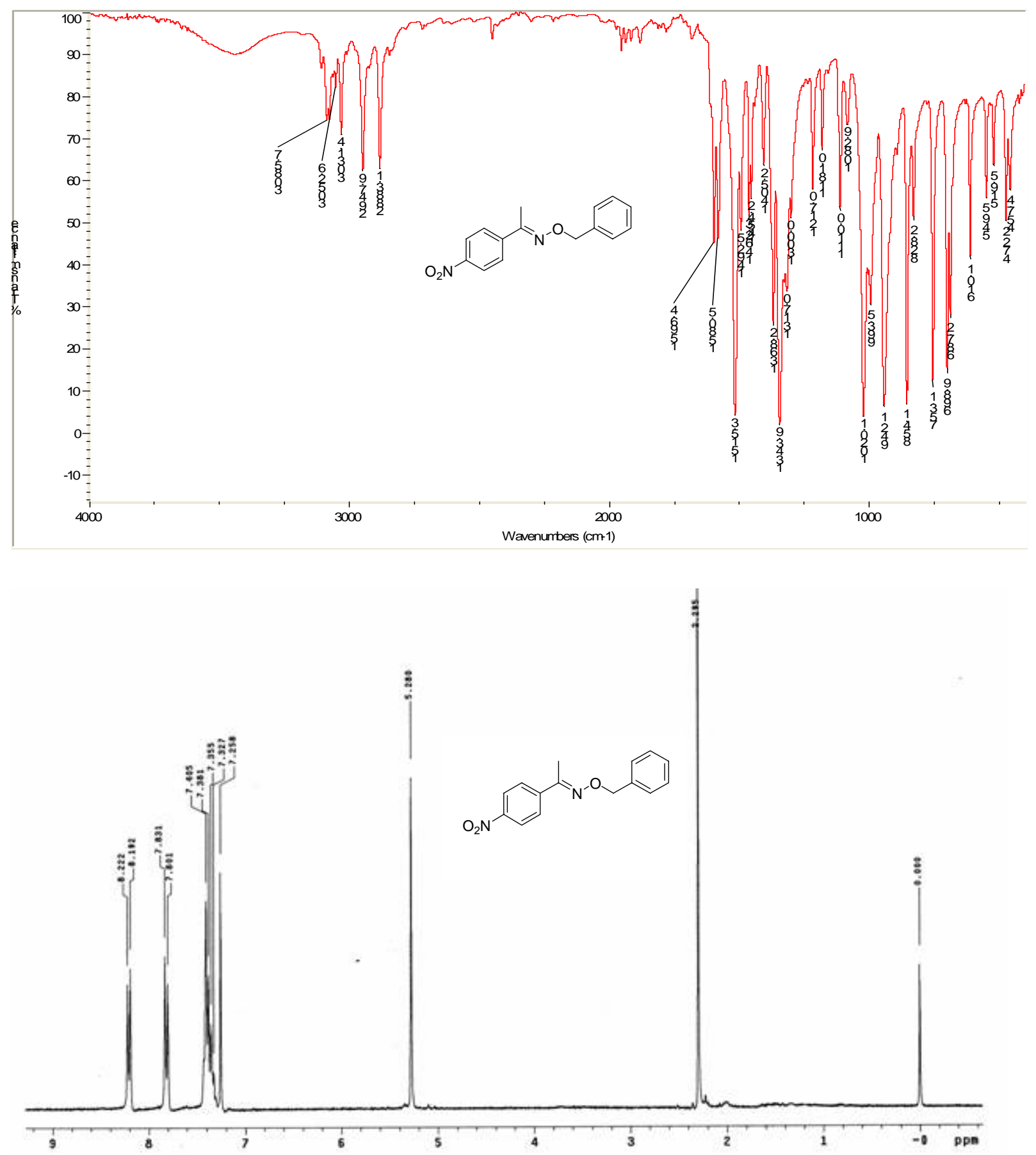

S22 

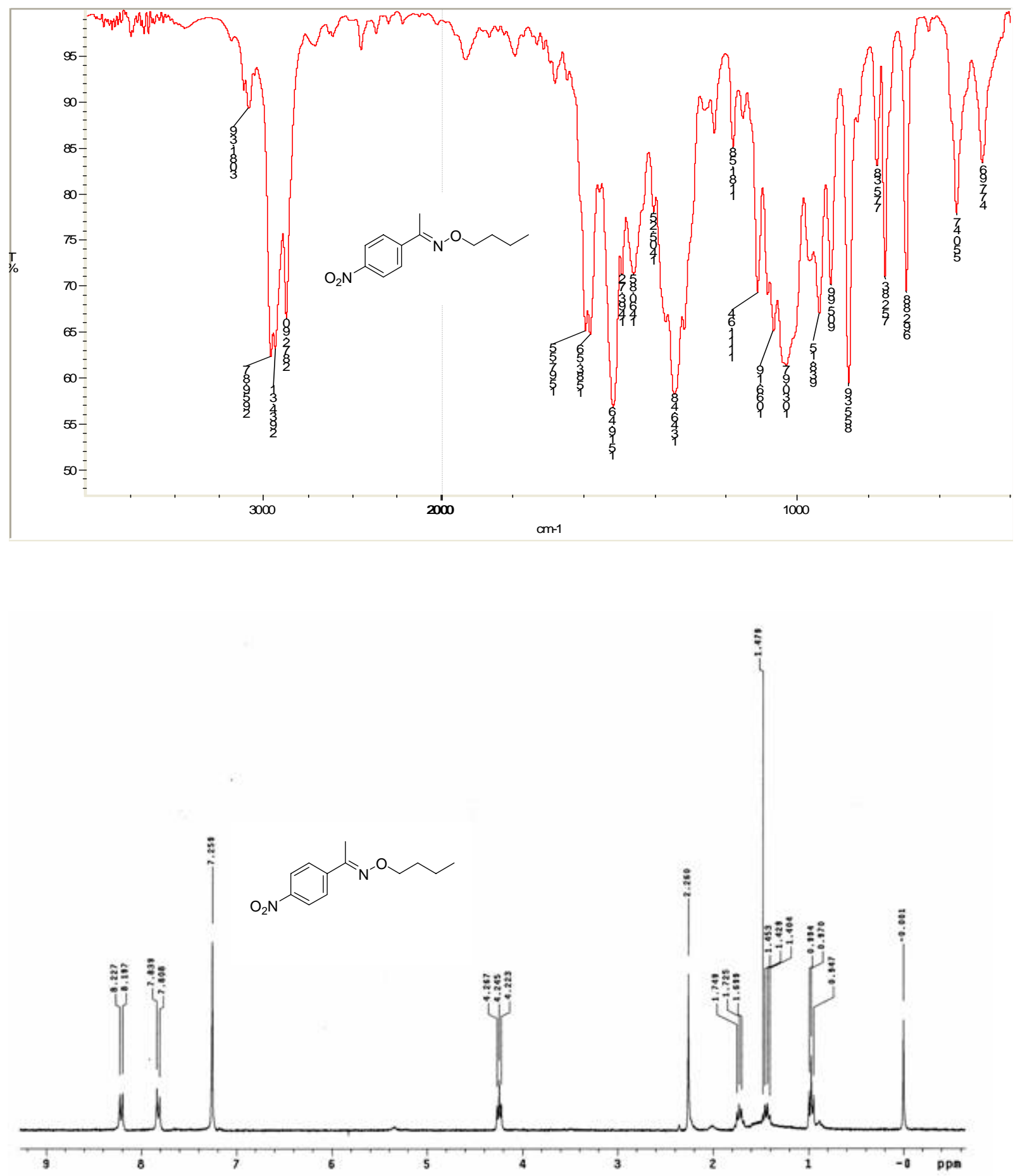

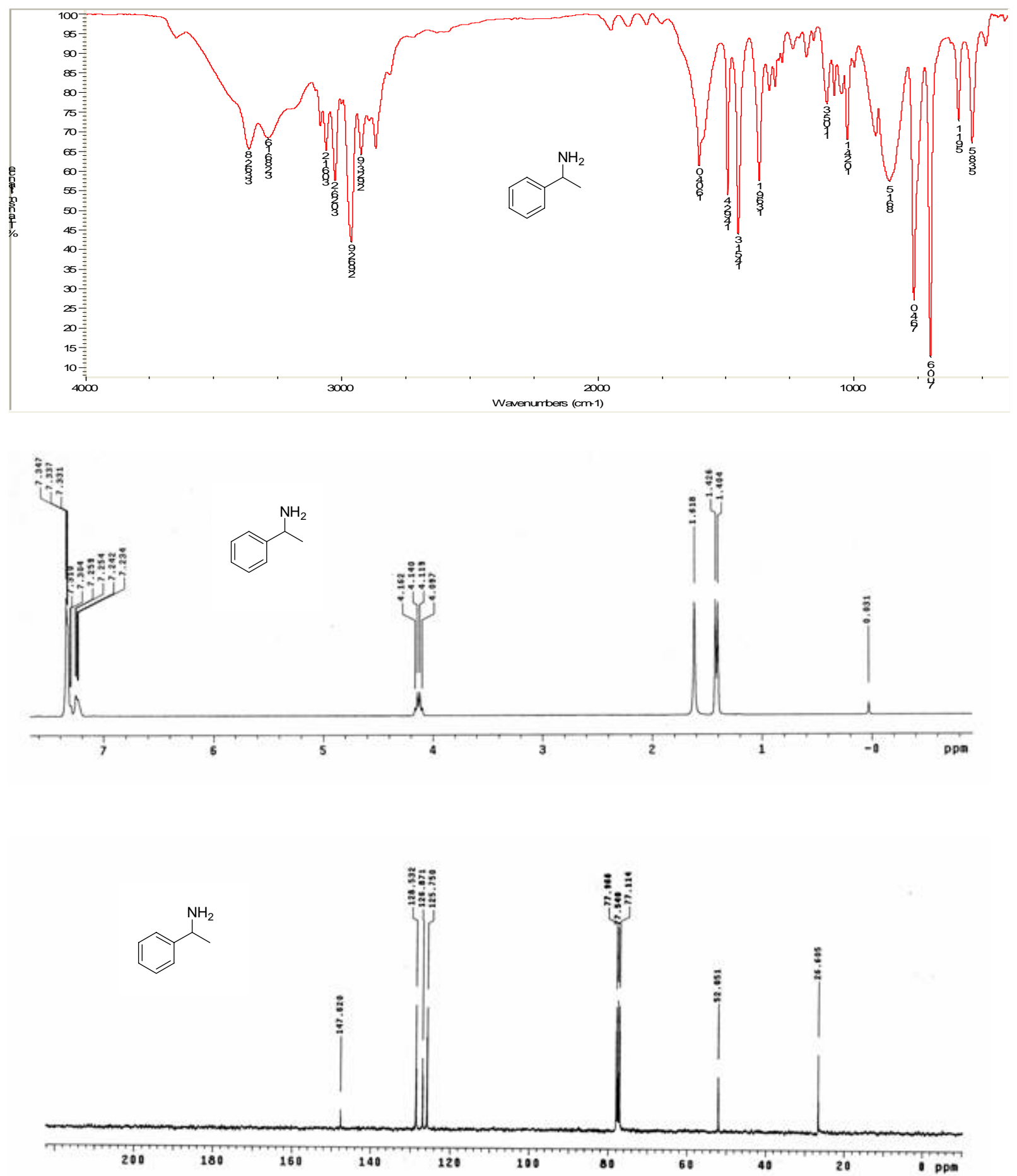

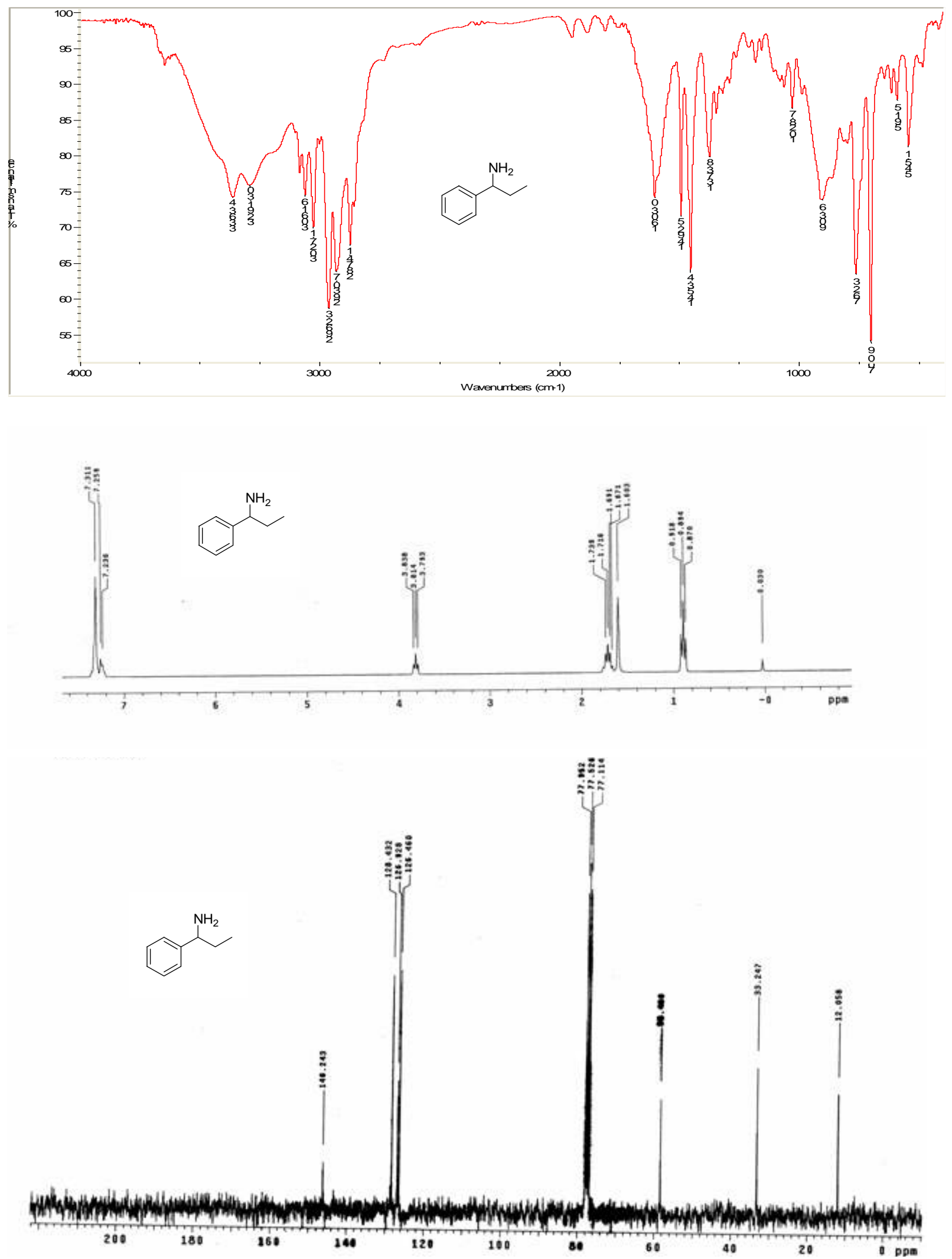

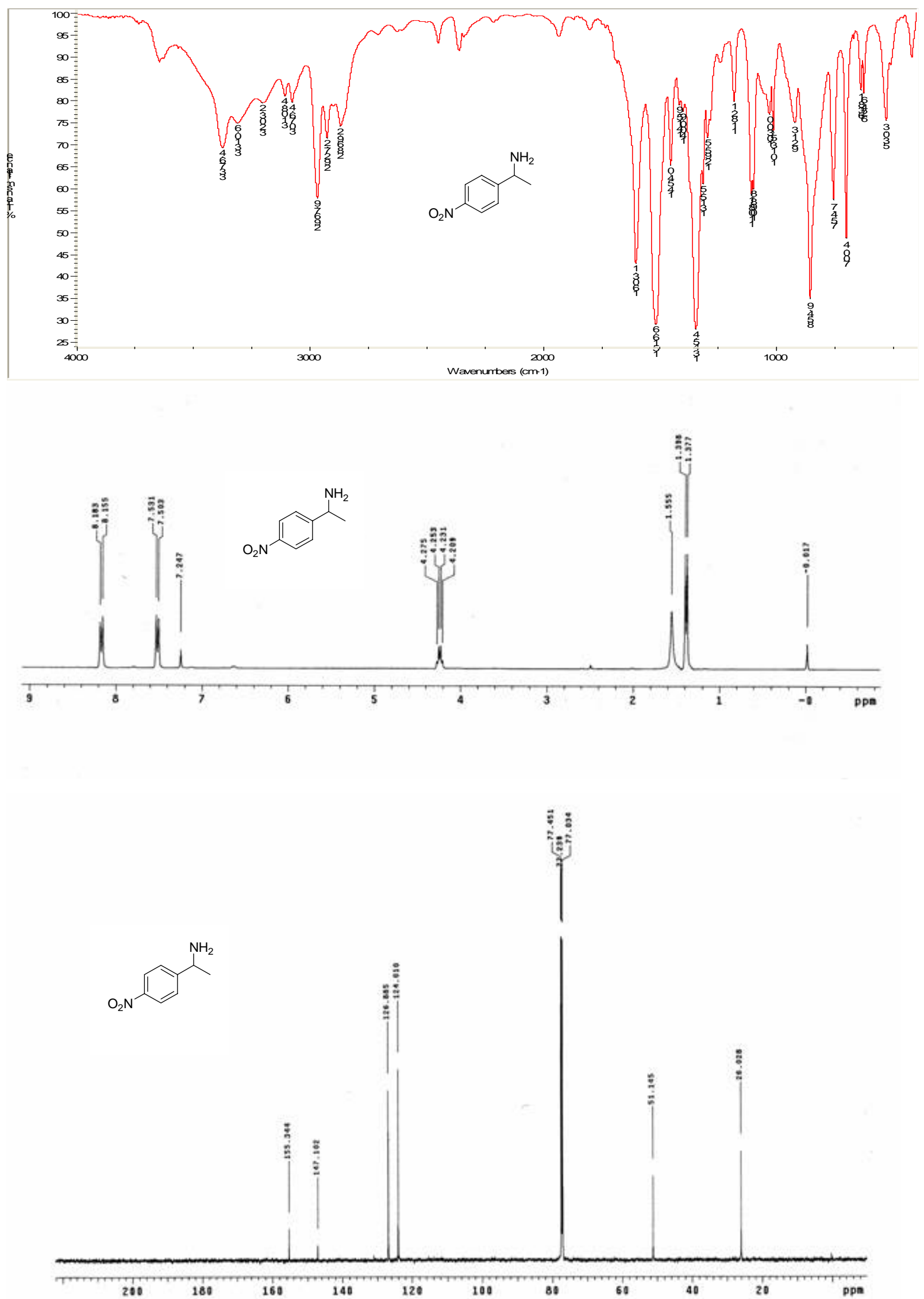

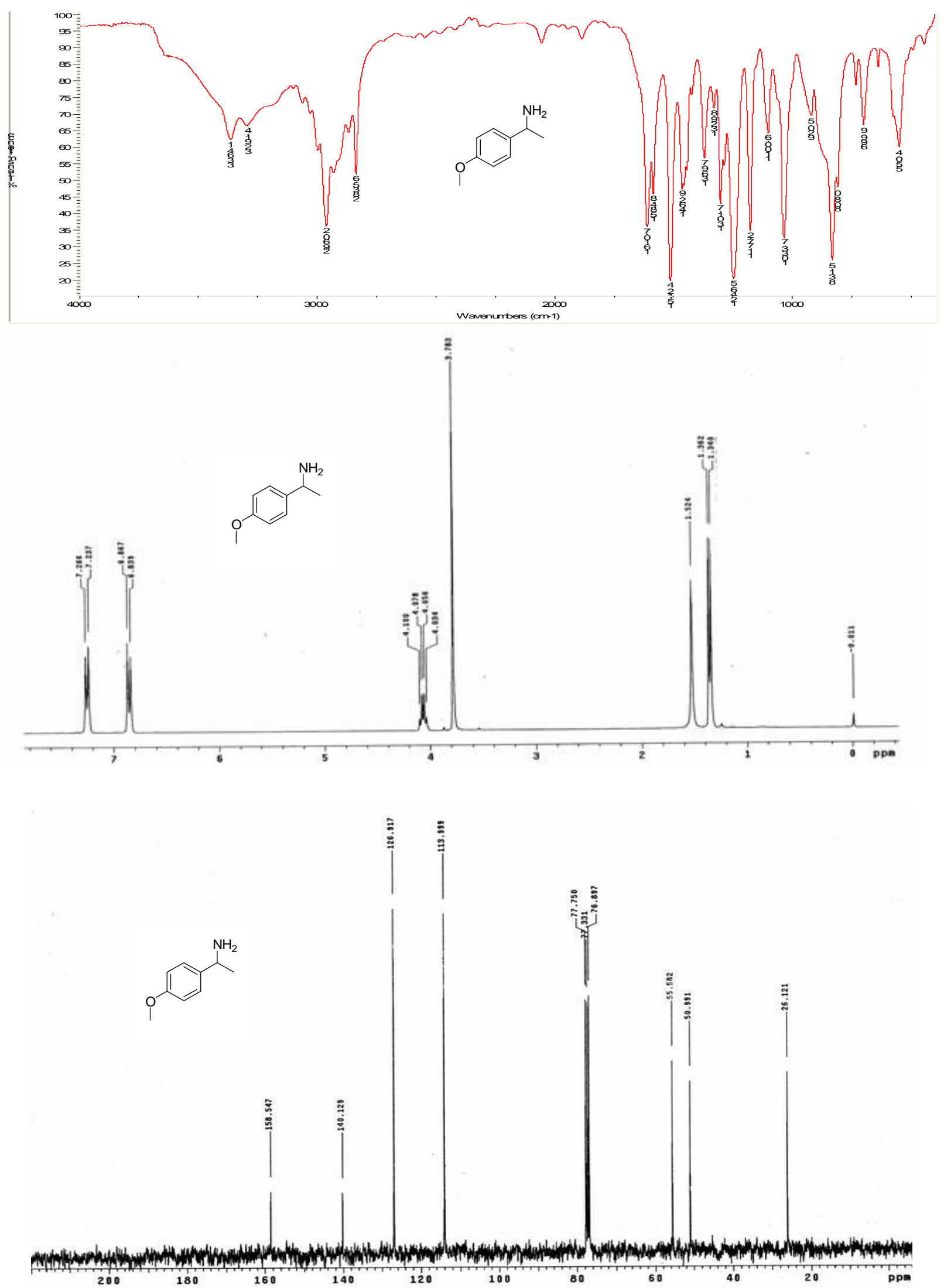

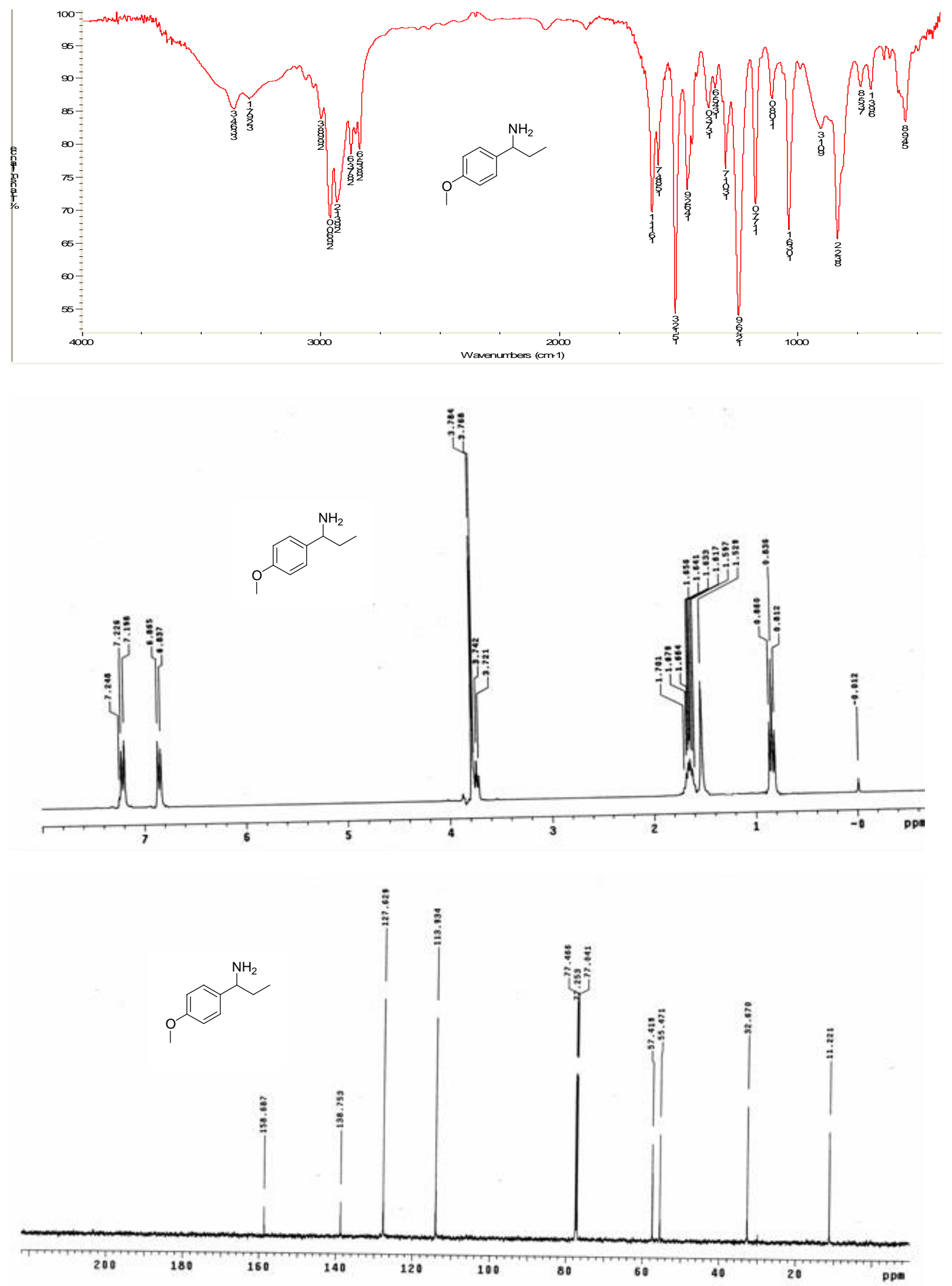

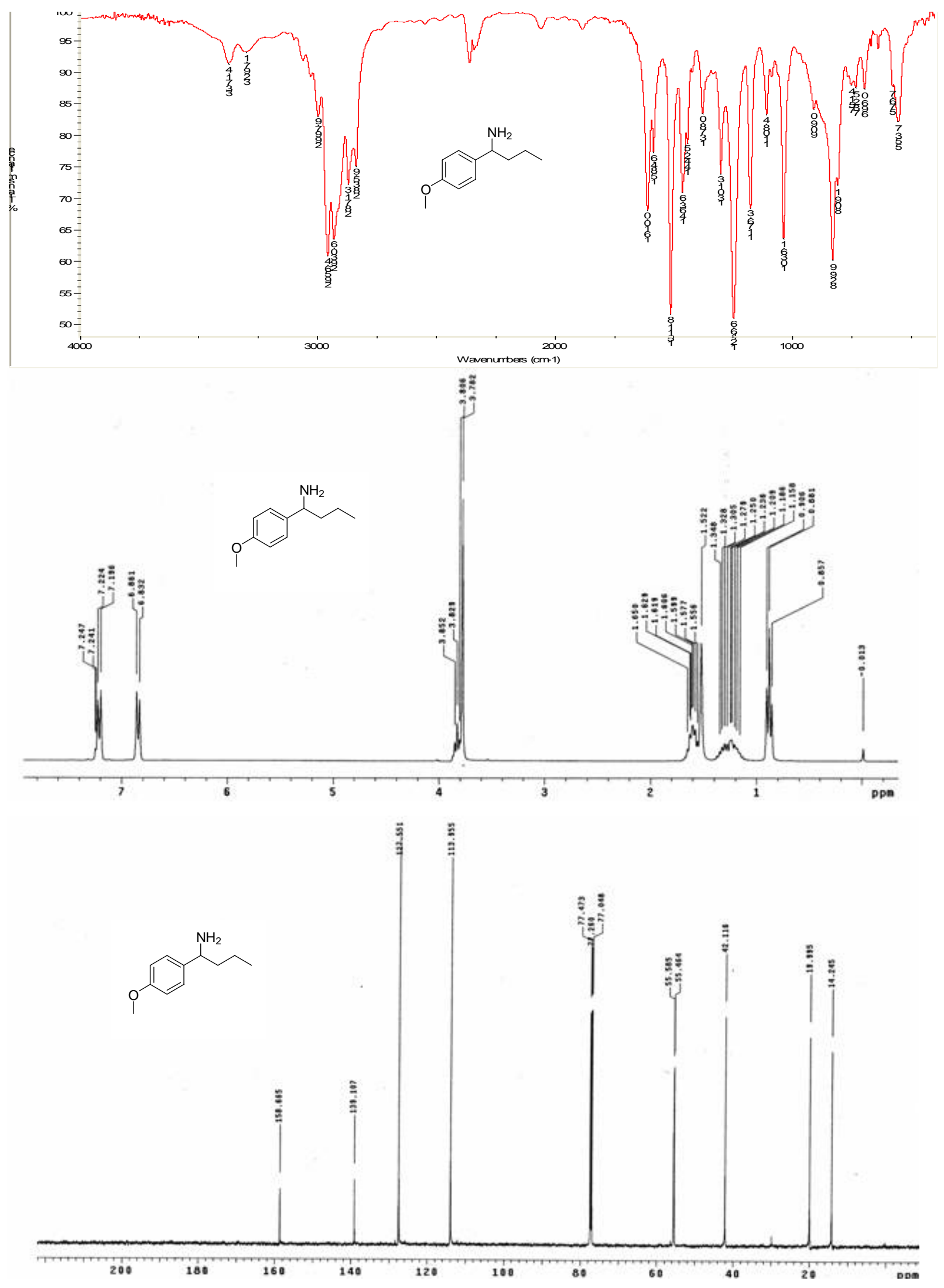
<smiles>CCCC(NC(C)=O)c1ccc(OC)cc1</smiles>

$( \pm)$

\begin{tabular}{|c|c|c|c|c|c|c|c|c|}
\hline Type & $\begin{array}{c}\text { Sanple } \\
\text { Nane }\end{array}$ & $\begin{array}{l}\text { Saaple } \\
\text { Amount }\end{array}$ & $\begin{array}{l}\text { Int Std } \\
\text { Anount }\end{array}$ & $\begin{array}{l}\text { Scale } \\
\text { factor }\end{array}$ & $\begin{array}{l}\mathrm{Nr} . \\
\text { Inj }\end{array}$ & $\begin{array}{l}\text { Vial } \\
\text { Mr. }\end{array}$ & $\begin{array}{l}\text { Inject } \\
\text { Vol ut }\end{array}$ & \\
\hline Unk & 30135849 & & & 100000 & 1 & & & fons 531 \\
\hline
\end{tabular}

\begin{tabular}{|c|c|c|c|c|c|c|c|c|c|c|c|}
\hline \multirow[t]{2}{*}{$\begin{array}{l}\text { Peak } \\
\text { Hunber } \\
\text { ::::::: }\end{array}$} & $\begin{array}{l}\text { Reteation } \\
\text { Tíe }\end{array}$ & $\begin{array}{l}\text { Couponent } \\
\text { Nase }\end{array}$ & $\begin{array}{c}\text { Concentration } \\
\mathrm{g} / \mathrm{at}\end{array}$ & $\begin{array}{l}\text { Morualized } \\
\text { Concentration }\end{array}$ & $\begin{array}{l}\text { Peak } \\
\text { Area }\end{array}$ & $\begin{array}{l}\text { Peak } \\
\text { Height }\end{array}$ & $\begin{array}{l}\text { Base } \\
\text { Code }\end{array}$ & $\begin{array}{l}\text { Response } \\
\text { Factor }\end{array}$ & $\begin{array}{l}\text { Rel. Ret } \\
\text { Tine }\end{array}$ & $\begin{array}{l}\text { Ares } \\
\text { Percent }\end{array}$ & $\begin{array}{l}\text { Meight } \\
\text { Percent }\end{array}$ \\
\hline & ::t:t::: & & ::::t:t:" & ::::::t:t:at: & :::::::::: & :m::t::: & ::::": & :::::::::: & :::::::: & :::::;: & ::::::: \\
\hline 1 & 0.326 & & 0.0000 & 0.000 & 0.02715 & 0.00049 & $B C V$ & 0.0000 & 0.0000 & 0.034 & 0.099 \\
\hline 2 & 0.397 & & 0.0000 & 0.000 & 0.01232 & 0.00058 & VCB & 0.0000 & 0.0000 & 0.016 & 0.115 \\
\hline 0 & 2.667 & & 0.0000 & 0.000 & 13.32660 & 0.20420 & BCB & 0.0000 & 0.0000 & 16.740 & 40.846 \\
\hline 4 & 3.925 & & 0.0000 & 0.000 & 0.29265 & 0.00115 & BNS & 0.0000 & 0.0000 & 0.367 & 0.230 \\
\hline 5 & 4.205 & & 0.0000 & 0.000 & 0.83721 & 0.00258 & SCB & 0.0000 & 0.0000 & 1.052 & 0.515 \\
\hline 6 & 5.592 & & 0.0000 & 0.000 & 32.22078 & 0.15237 & ACB & 0.0000 & 0.0000 & 40.474 & 30.479 \\
\hline 7 & 6.390 & & 0.0000 & 0.000 & 32.89188 & 0.13856 & $B C B$ & 0.0000 & 0.0000 & 41.317 & 27.716 \\
\hline \multirow{2}{*}{\multicolumn{3}{|c|}{$\begin{array}{l}\text { :::::: : } \\
\text { TOTALS }\end{array}$}} & :::::: & :::::: & 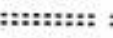 & & & & .1.0. & ::::::: & :::?:: \\
\hline & & & 0.0000 & 0.000 & 79.60859 & 0.49993 & & & & 100.000 & 100.000 \\
\hline
\end{tabular}

Average Efficiency: 0

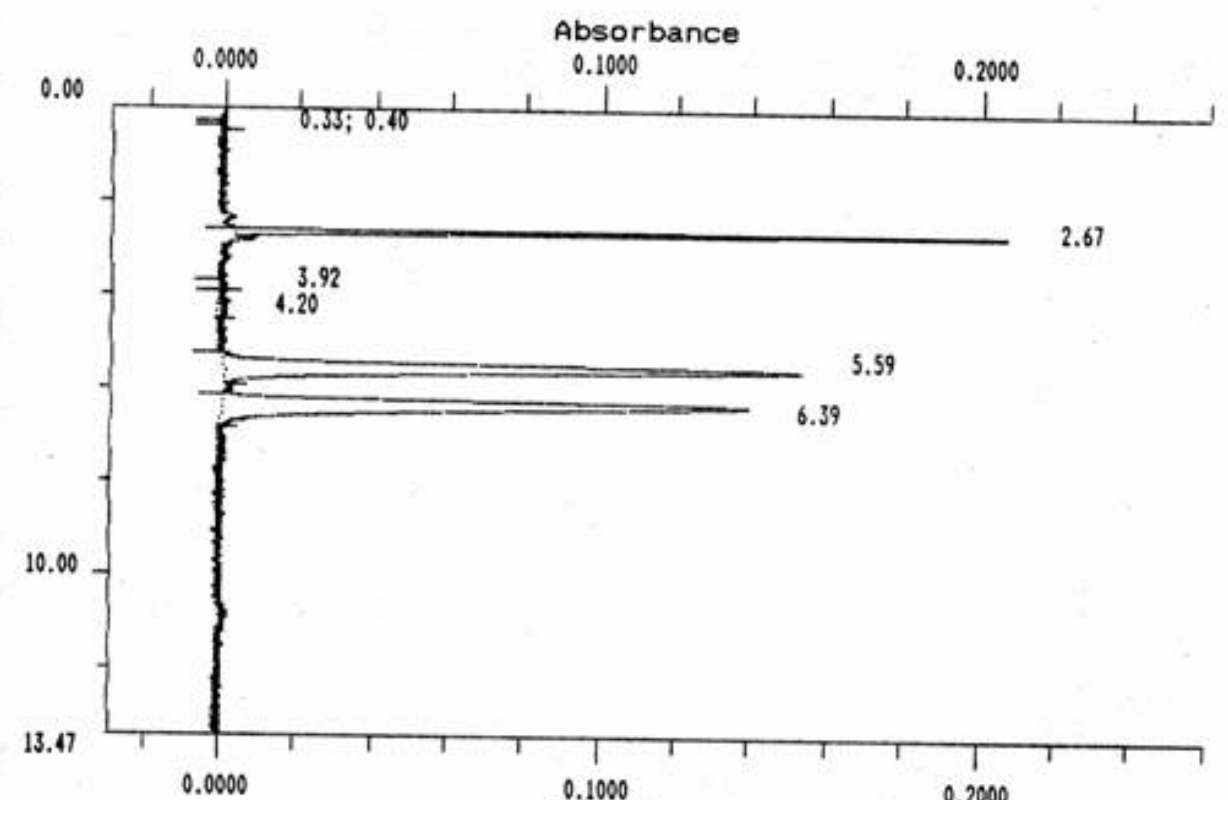

HPLC chromatogram for acetylated derivative of racemic 1-(4-methoxyphenyl)butylamine 


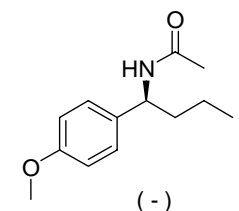

$(-)$

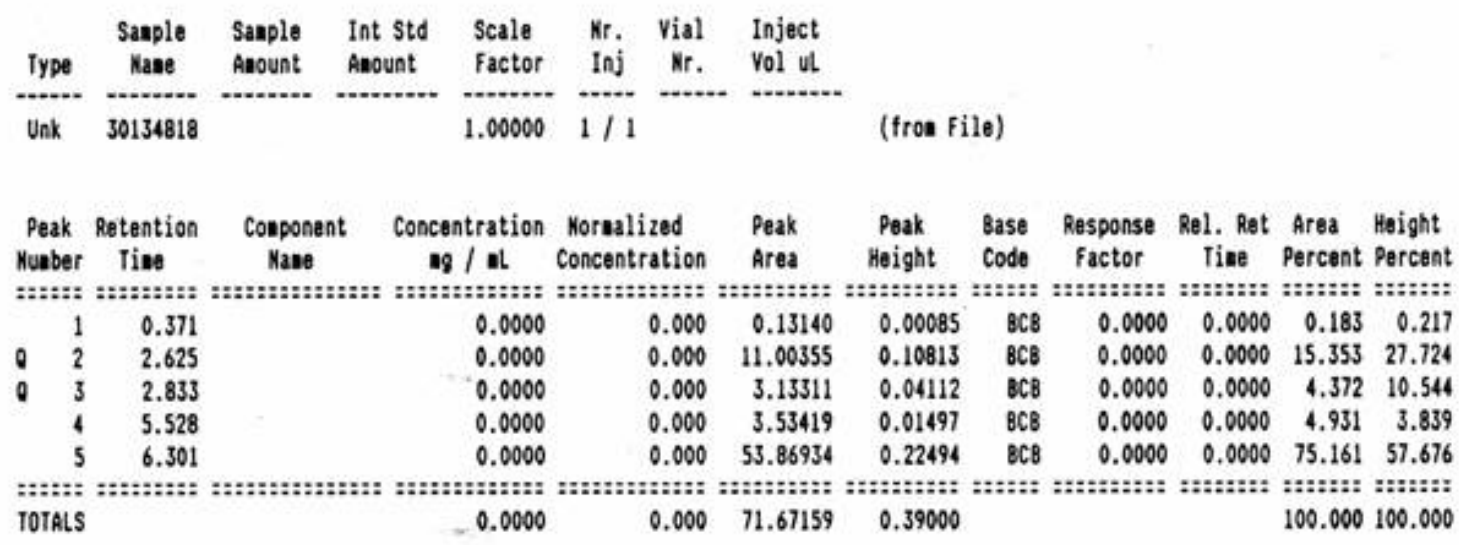

Average Efficiency: 0

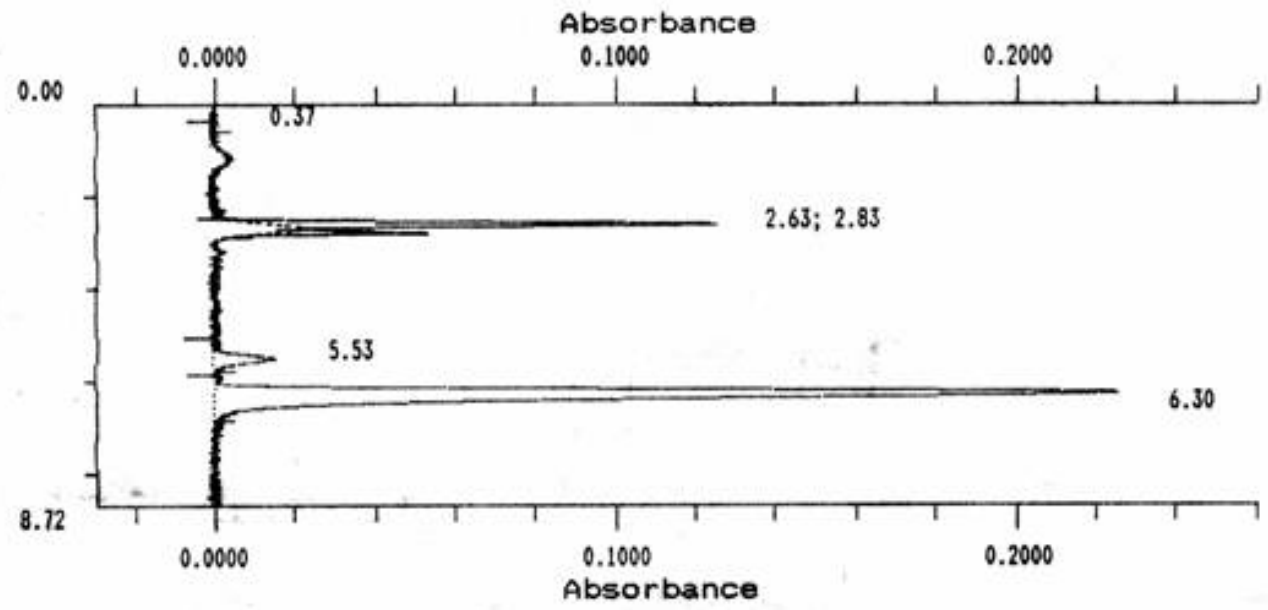

HPLC chramatogram for acetylated derivative of (S)-1-(4-methoxyphenyl)butylamine obtained from the reduction of O-methyl 4-methoxyphenyl propyl ketoxime 
<smiles>CCC(NC(C)=O)c1ccc(OC)cc1</smiles>

$( \pm)$

\begin{tabular}{ccccccccc} 
Type & $\begin{array}{c}\text { Sanple } \\
\text { Nane }\end{array}$ & $\begin{array}{c}\text { Sanple } \\
\text { Amount }\end{array}$ & $\begin{array}{l}\text { Int Std } \\
\text { Anount }\end{array}$ & $\begin{array}{l}\text { Scale } \\
\text { factor }\end{array}$ & $\begin{array}{l}\text { Mr. Vial } \\
\text { Inj }\end{array}$ Mr. & $\begin{array}{l}\text { Inject } \\
\text { Vol ut }\end{array}$ & \\
\hline Unk & 30131218 & & & 1.00000 & $1 / 1$ & & & (froe file)
\end{tabular}

Peak Retention Conponent Concentration Hornalized Peak Peak Base Response Rel. Ret Area Height Muaber Tine Mane o / at Concentration Area Height code factor tine Percent Percent

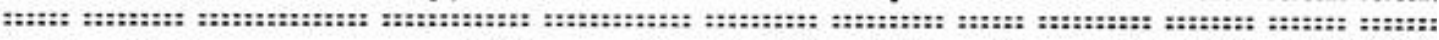
$\begin{array}{lllllllllllllll}0 & 1 & 2.675 & 0.0000 & 0.000 & 42.68852 & 0.59471 & \text { BCB } & 0.0000 & 0.0000 & 18.527 & 42.960\end{array}$

$\begin{array}{lllllllllll}2 & 5.721 & 0.0000 & 0.000 & 96.32253 & 0.42483 & \text { SCB } & 0.0000 & 0.0000 & 41.804 & 30.688\end{array}$

$\begin{array}{lllllllllllll}0 & 3 & 7.217 & 0.0000 & 0.000 & 91.40274 & 0.36481 & 8 C 8 & 0.0000 & 0.0000 & 39.669 & 26.352\end{array}$

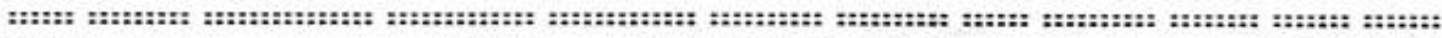
$\begin{array}{lllll}\text { TOTALS } & 0.0000 & 0.000 & 230.41379 & 1.38434\end{array}$

10000100.010

Average Efficiency: 0

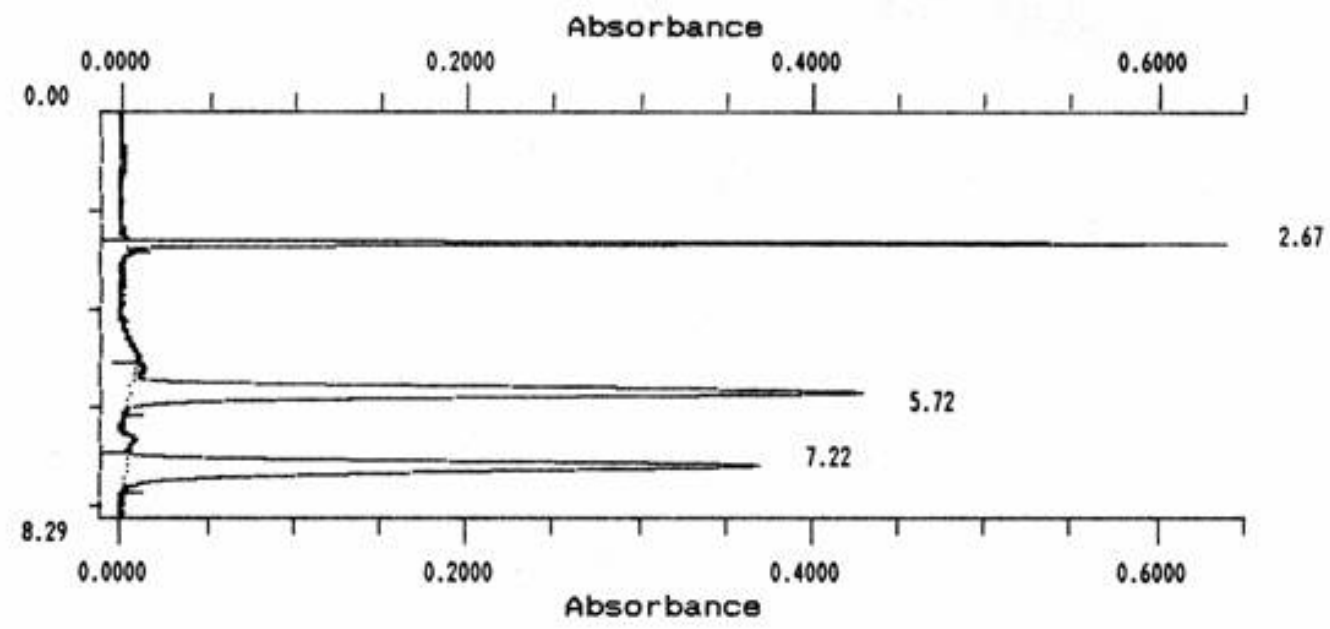

HPLC Chromatogram for acetylated derivative of racemic 1-(4-methoxyphenyl)propylamine 


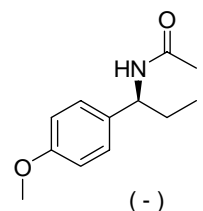

$(-)$

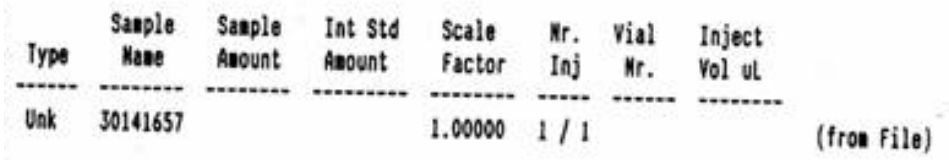

\begin{tabular}{|c|c|c|c|c|c|c|c|c|c|c|c|}
\hline $\begin{array}{l}\text { Peak } \\
\text { Nuaber }\end{array}$ & $\begin{array}{l}\text { Retention } \\
\text { Tine }\end{array}$ & $\begin{array}{c}\text { Couponent } \\
\text { Nane }\end{array}$ & $\begin{array}{c}\text { Concentration } \\
\text { a / al }\end{array}$ & $\begin{array}{l}\text { Noralized } \\
\text { Conceatration }\end{array}$ & $\begin{array}{l}\text { Peak } \\
\text { Area }\end{array}$ & $\begin{array}{l}\text { Peak } \\
\text { Height }\end{array}$ & $\begin{array}{l}\text { Base } \\
\text { Code }\end{array}$ & $\begin{array}{l}\text { Response } \\
\text { Factor }\end{array}$ & $\begin{array}{l}\text { Rel. Ret } \\
\text { Tíne }\end{array}$ & $\begin{array}{l}\text { Area } \\
\text { Percent }\end{array}$ & $\begin{array}{l}\text { Keight } \\
\text { Percent }\end{array}$ \\
\hline & $:::$ & & :::::::: & :::::a: & :::::m:z:: & ::::::t::s & :::::: & ::::::::a: & ::::::: & ::::::: & ::::::: \\
\hline 1 & 2.417 & & 0.0000 & 0.000 & $5.35 \% 65$ & 0.06672 & BCB & 0.0000 & 0.0000 & 10.163 & 25.901 \\
\hline 2 & 4.093 & & 0.0000 & 0.000 & 0.57727 & 0.00199 & $B C B$ & 0.0000 & 0.0000 & 1.095 & 0.773 \\
\hline 3 & 5.225 & & 0.0000 & 0.000 & 2.63119 & 0.00982 & $B C B$ & 0.0000 & 0.0000 & 4.991 & 3.813 \\
\hline 04 & 6.616 & & 0.0000 & 0.000 & 44.15081 & 0.17905 & BCB & 0.0000 & 0.0000 & 83.751 & 69.513 \\
\hline :::::2 & & & :: & & & ::::2::: & 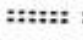 & :::::::::2 & ::::::: & ::::::: & : ::::::: \\
\hline TOTALS & & & 0.0000 & 0.000 & 52.71692 & 0.25757 & & & & 100.000 & 100.000 \\
\hline
\end{tabular}

Average Efficiency: 0

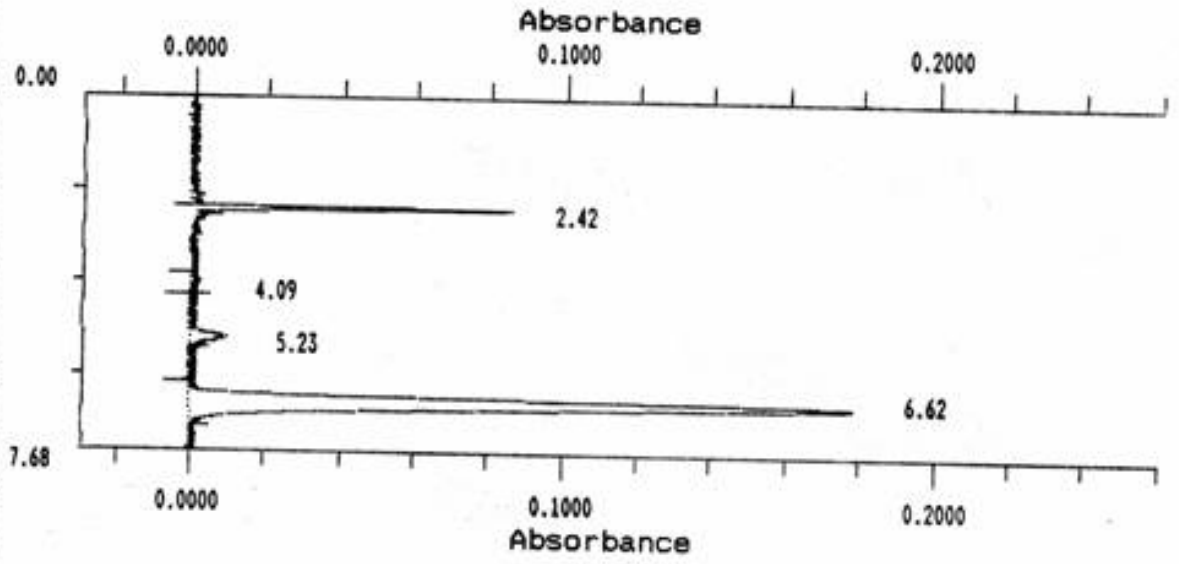

HPLC Chromatogram for acetylated derivative of (S)-1-(4-methoxyphenyl)propylamine obtained from the reduction of O-methyl 4-methoxyphenyl ethyl ketoxime 
<smiles>COc1ccc(C(C)NC(C)=O)cc1</smiles>

$( \pm)$

\begin{tabular}{ccccccccc} 
Type & $\begin{array}{c}\text { Sanple } \\
\text { Kane }\end{array}$ & $\begin{array}{c}\text { Saaple } \\
\text { Anount }\end{array}$ & $\begin{array}{l}\text { Int Std } \\
\text { Anount }\end{array}$ & $\begin{array}{l}\text { Scale } \\
\text { factor }\end{array}$ & $\begin{array}{l}\text { Inj } \\
\text { Inj }\end{array}$ & $\begin{array}{c}\text { Vial } \\
\text { An. }\end{array}$ & $\begin{array}{l}\text { Inject } \\
\text { Vol ut }\end{array}$ & \\
\hline Unk & 30123259 & & & 1.00000 & $1 / 1$ & & & (fron file)
\end{tabular}

\begin{tabular}{|c|c|c|c|c|c|c|c|c|c|c|c|}
\hline $\begin{array}{l}\text { Peak } \\
\text { Nunber }\end{array}$ & $\begin{array}{l}\text { Retention } \\
\text { Tive }\end{array}$ & $\begin{array}{l}\text { Coaponent } \\
\text { Mane }\end{array}$ & $\begin{array}{c}\text { Concentration } \\
\text { घg / at }\end{array}$ & $\begin{array}{l}\text { Morbalized } \\
\text { Concentration }\end{array}$ & $\begin{array}{l}\text { Peak } \\
\text { Area } \\
\end{array}$ & $\begin{array}{l}\text { Peak } \\
\text { Height }\end{array}$ & $\begin{array}{l}\text { 8ase } \\
\text { Code }\end{array}$ & $\begin{array}{l}\text { Response } \\
\text { Factor }\end{array}$ & $\begin{array}{l}\text { Rel. Ret } \\
\text { Tine }\end{array}$ & $\begin{array}{l}\text { Area } \\
\text { Percent }\end{array}$ & $\begin{array}{l}\text { Meight } \\
\text { Percent }\end{array}$ \\
\hline & & & & & & ::m::: & $::::$ & :::::::::: & :a::z:: & & \\
\hline 1 & 0.529 & & 0.0000 & 0.000 & 0.05639 & 0.00035 & $\mathrm{BCB}$ & 0.0000 & 0.0000 & 0.013 & 0.014 \\
\hline \multirow[t]{4}{*}{0} & 2.567 & & 0.0000 & 0.000 & 83.1 & 07 & $B C B$ & 0.0000 & 0.0000 & 18.941 & 45.096 \\
\hline & 4.098 & & 0.0000 & 0.000 & 2.58745 & 0.00826 & $B C B$ & 0.0000 & 0.0000 & 0.590 & 0.334 \\
\hline & 4.940 & & 0.0000 & 0.000 & 1.24920 & 0.00712 & $B C B$ & 0.0000 & 0.0000 & 0.285 & 0.289 \\
\hline & 5.886 & & 0.0000 & 0.000 & 176.97403 & 881 & $B C B$ & 000 & 0.0000 & 40.321 & 28.367 \\
\hline 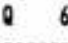 & 7.150 & & 0.0000 & 0.000 & 218 & 3985 & BCB & 0.0000 & 0.0000 & 39.850 & 25.900 \\
\hline \multirow{2}{*}{\multicolumn{2}{|c|}{ TOTALS }} & & & & & & & & & (1) & 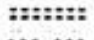 \\
\hline & & & 0.0000 & 0.000 & 438.90494 & 2.47047 & & & & 100.0001 & 100.000 \\
\hline
\end{tabular}

Average Efficiency: 0

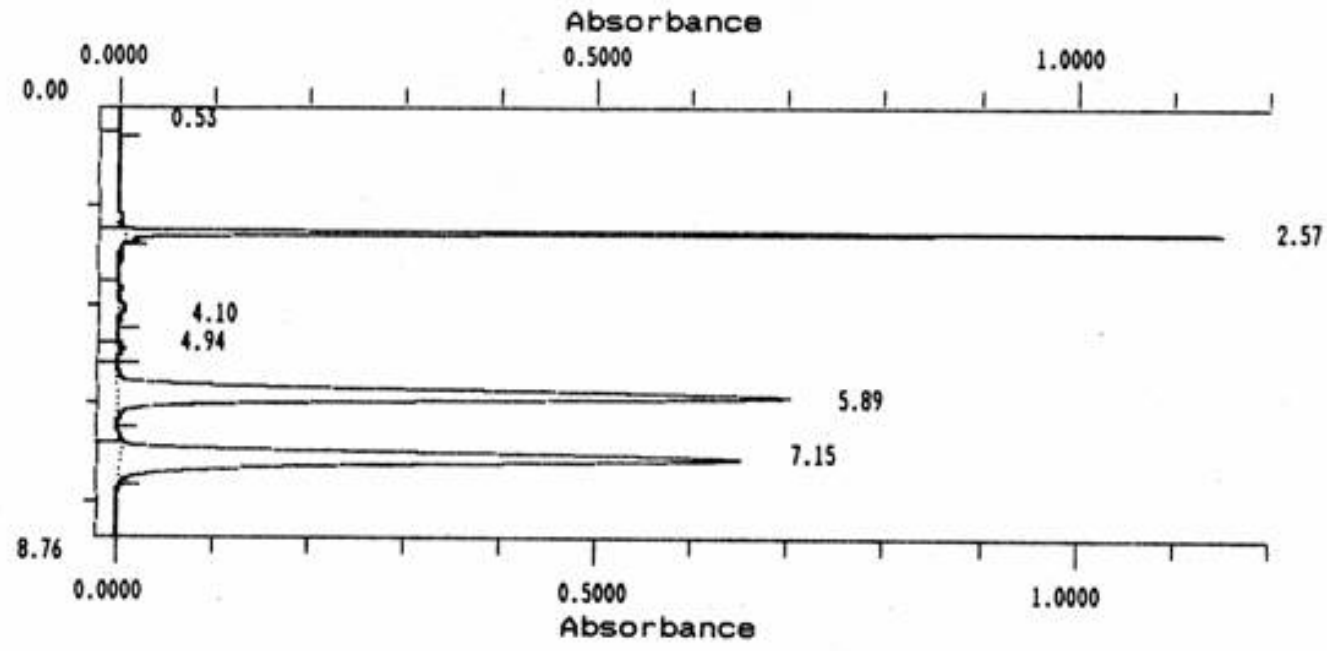

HPLC chromatogram for acetylated derivative of racemic 1-(4-methoxyphenyl)ethylamine 
<smiles>COc1ccc(C(C)NC(C)=O)cc1</smiles>

\begin{tabular}{|c|c|c|c|c|c|c|c|}
\hline Type & $\begin{array}{l}\text { Sanple } \\
\text { Nane }\end{array}$ & $\begin{array}{l}\text { Sanple } \\
\text { Anounat }\end{array}$ & $\begin{array}{l}\text { Int Std } \\
\text { Aacount }\end{array}$ & $\begin{array}{l}\text { Scale } \\
\text { factor }\end{array}$ & $\begin{array}{l}\text { Mr. } \\
\text { Inj }\end{array}$ & $\begin{array}{l}\text { Vial } \\
\text { Mr. }\end{array}$ & $\begin{array}{l}\text { Inject } \\
\text { Vol ut }\end{array}$ \\
\hline & 30125300 & & & 1.00000 & $1 / 1$ & & \\
\hline
\end{tabular}

\begin{tabular}{|c|c|c|c|c|c|c|c|c|c|c|c|}
\hline $\begin{array}{l}\text { Peak } \\
\text { Nuaber }\end{array}$ & $\begin{array}{l}\text { Rateation } \\
\text { Tine }\end{array}$ & $\begin{array}{l}\text { Couponent } \\
\text { Nane }\end{array}$ & $\begin{array}{c}\text { Concentration } \\
\text { g / al }\end{array}$ & $\begin{array}{l}\text { Moraalized } \\
\text { Concentration }\end{array}$ & $\begin{array}{l}\text { Peak } \\
\text { Area }\end{array}$ & $\begin{array}{l}\text { Peak } \\
\text { Height }\end{array}$ & $\begin{array}{l}\text { Base } \\
\text { code }\end{array}$ & $\begin{array}{l}\text { Response } \\
\text { factor }\end{array}$ & $\begin{array}{l}\text { Rel. Ret } \\
\text { Tine }\end{array}$ & $\begin{array}{l}\text { Area } \\
\text { Percent }\end{array}$ & $\begin{array}{l}\text { Height } \\
\text { Percent }\end{array}$ \\
\hline & .... & ::: & :::::::: & :z:::::::::t:: & $::$ & $\because 2$ & it & H:H: & 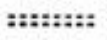 & ::: & : \\
\hline 1 & 0.257 & & 0.0000 & 0.000 & 0.00601 & 0.00046 & $B C B$ & 0.0000 & 0.0000 & 0.003 & 0.059 \\
\hline 2 & 2.675 & & 0.0000 & 0.000 & 7.32024 & 0.11097 & $\mathrm{BCB}$ & 0.0000 & 0.0000 & 3.937 & 14.266 \\
\hline 3 & 6.107 & & 0.0000 & 0.000 & 8.60447 & 0.03615 & $B C B$ & 0.0000 & 0.0000 & 4.628 & 4.648 \\
\hline 4 & 7.442 & & 0.0000 & 0.000 & 170.00163 & 0.63027 & BCB & 0.0000 & 0.0000 & 91.432 & 81.027 \\
\hline TOTALS & ....... & & 0.0000 & 0,000 & 185.93235 & היורי & 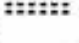 & & & $100 \mathrm{Mm}$ & $10 m \mathrm{~mm}$ \\
\hline
\end{tabular}

Average Efficiency: 0

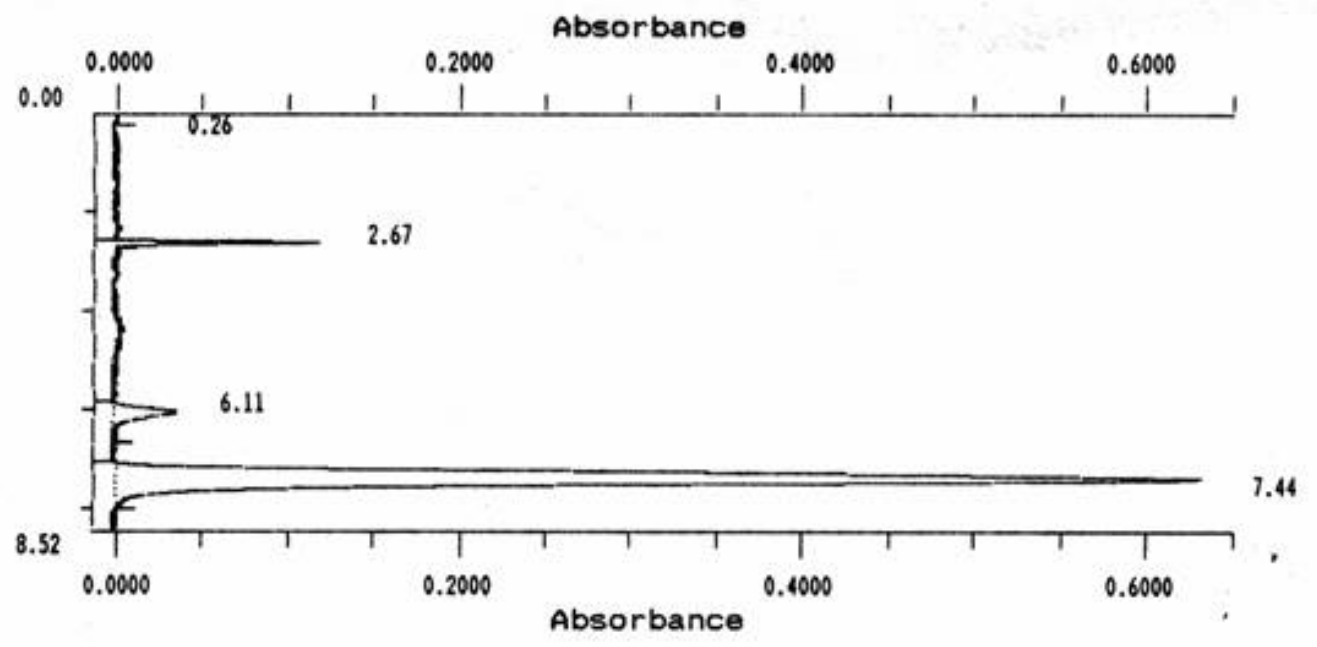

HPLC chromatogram for acetylated derivative of $(S)$-1-(4-methoxyphenyl)ethylamine obtained from the reduction of O-methyl 4-methoxyphenyl methyl ketoxime 


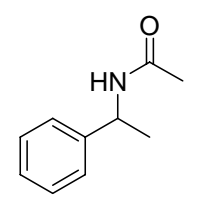

(土)

SAMPLE TABLE nONe

SYSTEM 1: SYSTEMI

\section{Chart Speed $0.50 \mathrm{ca} / \mathrm{hin}$}

\begin{tabular}{ccccccccc} 
Type & $\begin{array}{c}\text { Sanple } \\
\text { Nase }\end{array}$ & $\begin{array}{c}\text { Sanple } \\
\text { Anount }\end{array}$ & $\begin{array}{l}\text { Int Std } \\
\text { Anount }\end{array}$ & $\begin{array}{l}\text { Scale } \\
\text { Factor }\end{array}$ & $\begin{array}{c}\text { Mr. } \\
\text { Inj }\end{array}$ & $\begin{array}{c}\text { Kial } \\
\text { Mr. }\end{array}$ & $\begin{array}{l}\text { Inject } \\
\text { Vol ut }\end{array}$ & \\
\hline Unk & 02091035 & & & 1.00000 & $1 / 1$ & & & (frou file)
\end{tabular}

\begin{tabular}{|c|c|c|c|c|c|c|c|c|c|c|c|}
\hline $\begin{array}{l}\text { Peak } \\
\text { Munber }\end{array}$ & $\begin{array}{l}\text { Retention } \\
\text { Tine }\end{array}$ & $\begin{array}{c}\text { Conponent } \\
\text { Nane }\end{array}$ & $\begin{array}{c}\text { Concentration } \\
\text { g / }\end{array}$ & $\begin{array}{l}\text { Moralized } \\
\text { Concentration }\end{array}$ & $\begin{array}{l}\text { Peak } \\
\text { Area }\end{array}$ & $\begin{array}{l}\text { Peak } \\
\text { Meight }\end{array}$ & $\begin{array}{l}\text { Base } \\
\text { code }\end{array}$ & $\begin{array}{l}\text { Response } \\
\text { Factor } \\
\text { :a::::: }\end{array}$ & $\begin{array}{l}\text { Rel. Ret } \\
\text { Tine } \\
\text { :::::::: }\end{array}$ & $\begin{array}{l}\text { Area } \\
\text { Percent } \\
\text { :z:z:z: }\end{array}$ & $\begin{array}{l}\text { Height } \\
\text { Percent } \\
\text { ::::::: }\end{array}$ \\
\hline$\cdots$ & 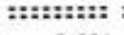 & $\cdots$ & mat:: & :::::: & a:z:a:: & ::::::::: & & & $::::$ & 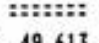 & $=$ \\
\hline 1 & 3.936 & & 0.0000 & 0.000 & 30.00108 & 0.14309 & $\begin{array}{l}8 C B \\
B C B\end{array}$ & $\begin{array}{l}0.0000 \\
0.0000\end{array}$ & $\begin{array}{l}0.0000 \\
0.0000\end{array}$ & $\begin{array}{l}49.613 \\
50.387\end{array}$ & .060 \\
\hline 2 & 4.677 & & 0.0000 & $\begin{array}{r}0.000 \\
::::::=\end{array}$ & $\begin{array}{r}30.46896 \\
::::::::: 2\end{array}$ & a:15/15 & :::::: & ::::::::: & .... & ::::::: & \\
\hline TAL & & & 0.0000 & 0.000 & 60.47004 & 0.28025 & & & & 100.000 & 100.0 \\
\hline
\end{tabular}

Average Efficiency: 0

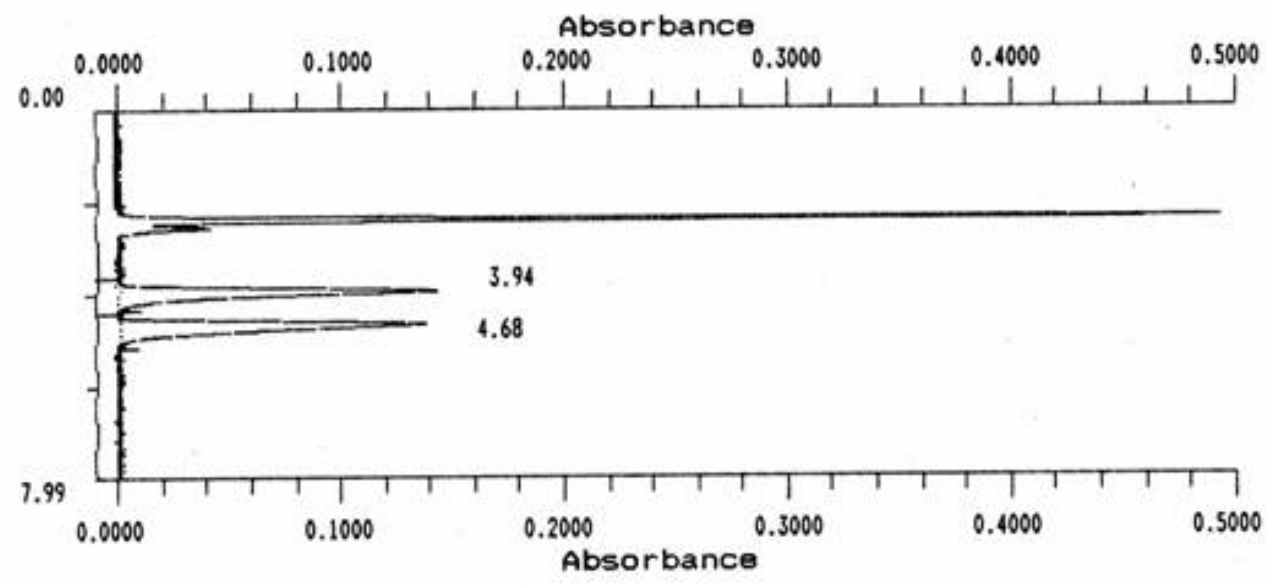

HPLC chromatogram for acetylated derivative of racemic 1-phenylethylamine 
<smiles>CC(=O)NC(C)c1ccccc1</smiles>

$(-)$

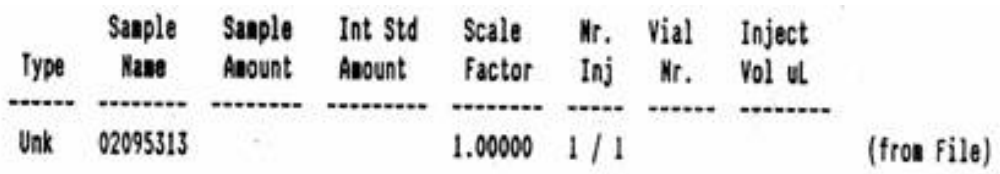

\begin{tabular}{|c|c|c|c|c|c|c|c|c|c|c|c|}
\hline $\begin{array}{c}\text { Poak } \\
\text { Nuaber }\end{array}$ & $\begin{array}{l}\text { Retention } \\
\text { Tine }\end{array}$ & $\begin{array}{c}\text { Conponent } \\
\text { Nane }\end{array}$ & $\begin{array}{c}\text { Concentration } \\
\text { ng / al }\end{array}$ & $\begin{array}{l}\text { Morealized } \\
\text { Concentration }\end{array}$ & $\begin{array}{l}\text { Peak } \\
\text { Area }\end{array}$ & $\begin{array}{l}\text { Peak } \\
\text { Meight }\end{array}$ & $\begin{array}{l}\text { Base } \\
\text { Code }\end{array}$ & $\begin{array}{l}\text { Response } \\
\text { Factor. }\end{array}$ & $\begin{array}{l}\text { Rel. Ret } \\
\text { Tine }\end{array}$ & $\begin{array}{l}\text { Area } \\
\text { Percent }\end{array}$ & $\begin{array}{l}\text { Height } \\
\text { Percent }\end{array}$ \\
\hline ... & & & & & :::::::::: & - & . & & ( & & \\
\hline 0 & 2.500 & & 0.0000 & 0.000 & 133.61227 & 1.48282 & $B C B$ & 0.0000 & 0.0000 & 4.243 & 69.277 \\
\hline 2 & 4.172 & & 0.0000 & 0.000 & 9.47655 & 0.04745 & $B C B$ & 0.0000 & 0.0000 & 3.139 & 2.217 \\
\hline 3 & 4.857 & & 0.0000 & 0.000 & 158.56706 & 0.60997 & BCS & 0.0000 & 0.0000 & 52.506 & 28.497 \\
\hline 4 & 6.033 & & 0.0000 & 0.000 & 0.33713 & 0.00019 & SHB & 0.0000 & 0.0000 & 0.112 & 0.009 \\
\hline & & & iniin & & & & & w.... & ::::::: & :::::: & \\
\hline TOTALS & & & 0.0000 & 0.000 & 301.99302 & 2.14043 & & & & 100.000 & \\
\hline
\end{tabular}

Average Efficiency: 0

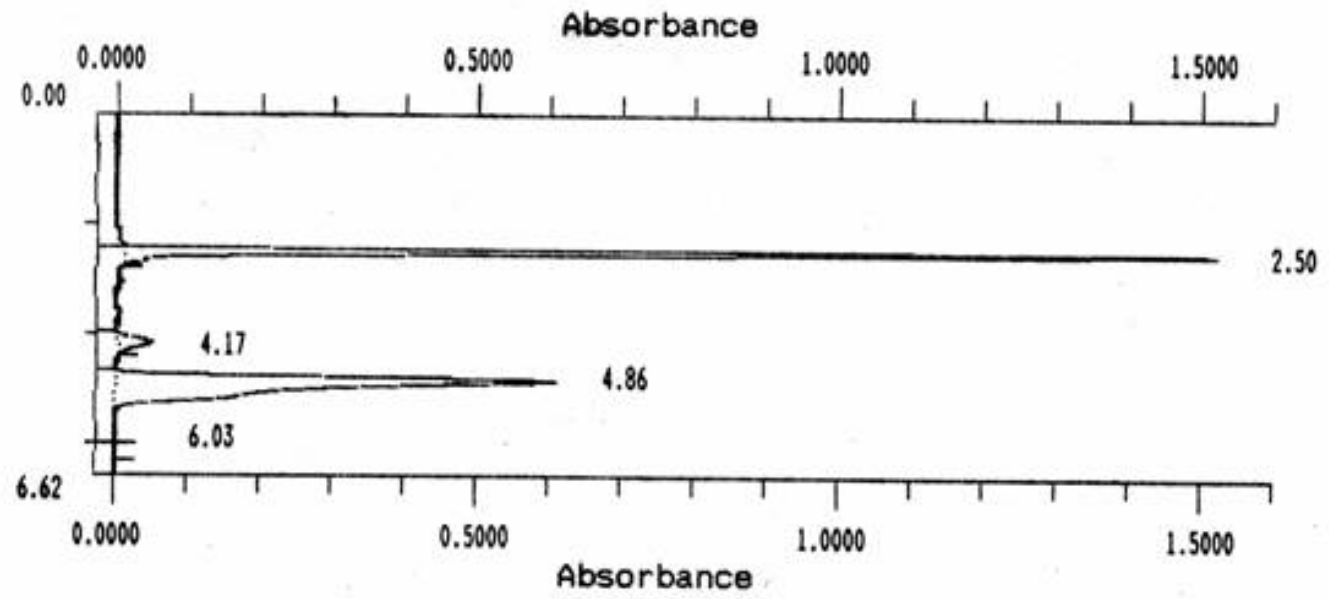

HPLC chromatogram for acetylated derivative of $(S)$-1-phenylethylamine obtained from the reduction of O-benzyl acetophenoneoxime 
<smiles>CC(=O)NC(C)c1ccccc1</smiles>

( - )

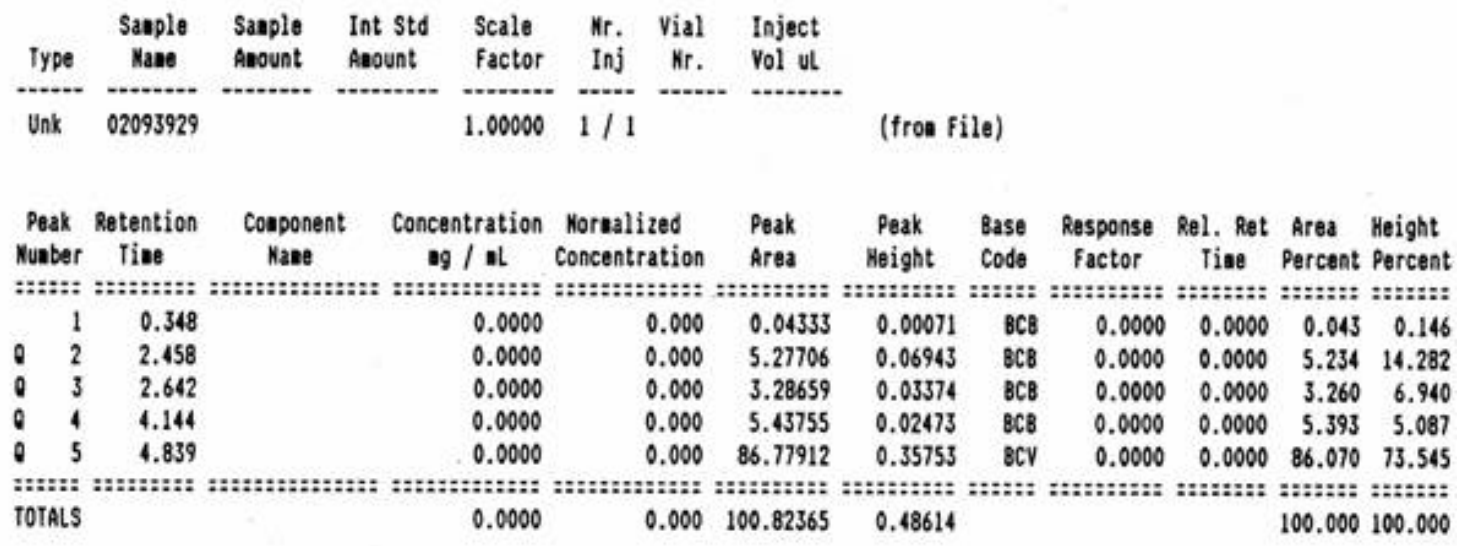

Average Efficiency: 0

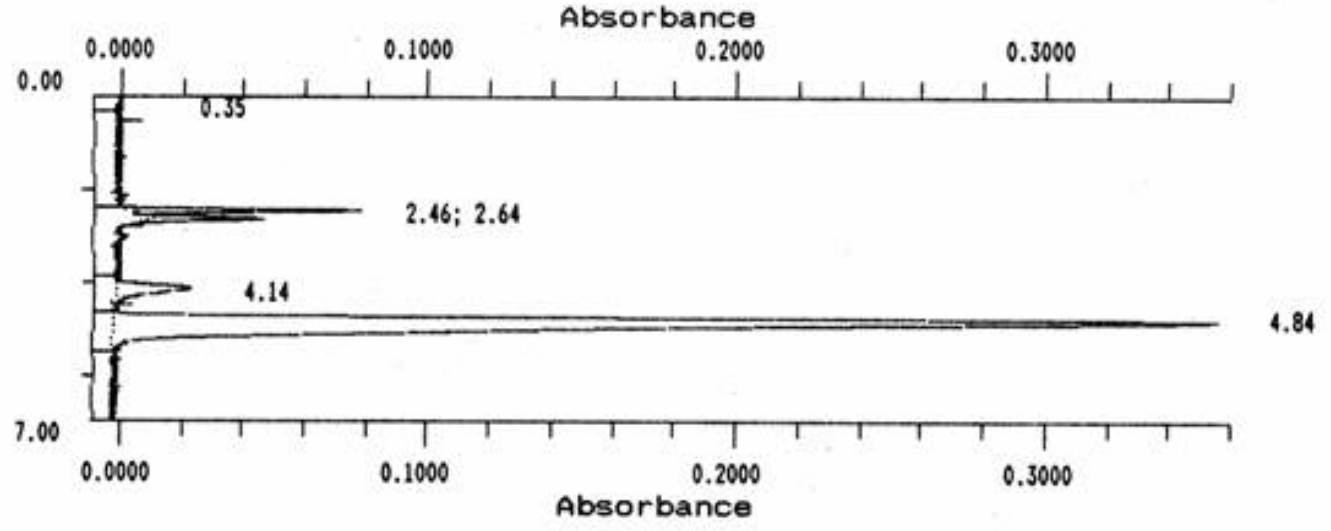

HPLC chromatogram for acetylated derivative of (S)-1-phenylethylamine obtained from the reduction of $O$-methyl acetophenoneoxime 


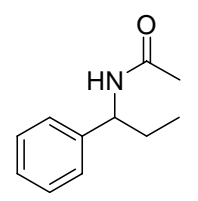

$( \pm)$

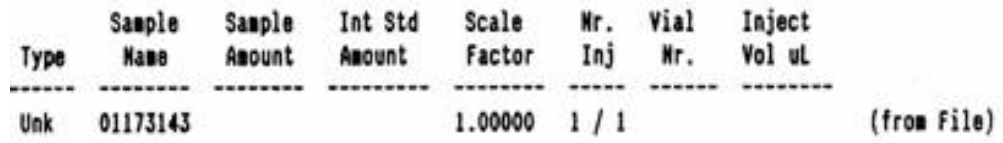

\begin{tabular}{|c|c|c|c|c|c|c|c|c|c|c|c|}
\hline $\begin{array}{l}\text { Peak } \\
\text { Nuaber } \\
\text { :::::: }\end{array}$ & $\begin{array}{l}\text { Retention } \\
\text { Tine } \\
\text { ::::::::: }\end{array}$ & $\begin{array}{l}\text { Conponent } \\
\text { Nane } \\
\text { ::t::t:t::: }\end{array}$ & 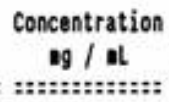 & $\begin{array}{l}\text { Norualized } \\
\text { Concentration } \\
\text { :::z:z:z:z::z }\end{array}$ & $\begin{array}{l}\text { Peak } \\
\text { Area } \\
\text { ::::::::: }\end{array}$ & $\begin{array}{l}\text { Peak } \\
\text { Height } \\
\text { :::::::::Z: }\end{array}$ & $\begin{array}{l}\text { Base } \\
\text { Code } \\
\text { :::::: }\end{array}$ & $\begin{array}{l}\text { Response } \\
\text { Factor } \\
\text { :a:::s:::: }\end{array}$ & $\begin{array}{l}\text { Rel. Ret } \\
\text { Tine } \\
\text { ::t::::: }\end{array}$ & $\begin{array}{l}\text { Area } \\
\text { Percent } \\
\text { :zez:a: }\end{array}$ & $\begin{array}{l}\text { Height } \\
\text { Percent } \\
\text { :z:z:t: }\end{array}$ \\
\hline 1 & 0.566 & & 0.0000 & 0.000 & 0.03076 & 0.00092 & $B C B$ & 0.0000 & 0.0000 & 0.029 & 0.175 \\
\hline 2 & 3.871 & & 0.0000 & 0.000 & 50.83171 & 0.26368 & $B C B$ & 0.0000 & 0.0000 & 48.456 & 50.216 \\
\hline 3 & 4.638 & & 0.0000 & 0.000 & 53.12435 & 0.25824 & $B C B$ & 0.0000 & 0.0000 & 50.642 & 49.182 \\
\hline 4 & 5.894 & & .0000 & 0.000 & 48372 & 0.00137 & $B C B$ & 0.0000 & 0.0000 & 0.461 & 0.262 \\
\hline 5 & 6.419 & & 0.0000 & 0.000 & 0.43204 & 0.00087 & $B C B$ & 0.0000 & 0.0000 & 0.412 & 0.165 \\
\hline & & & & & & & 1.0 & $\cdots$ & & & \\
\hline FALS & & & 0.0000 & 0.000 & 104.90258 & 0.52508 & & & & 100.000 & 100.000 \\
\hline
\end{tabular}

Average Efficiency: 0

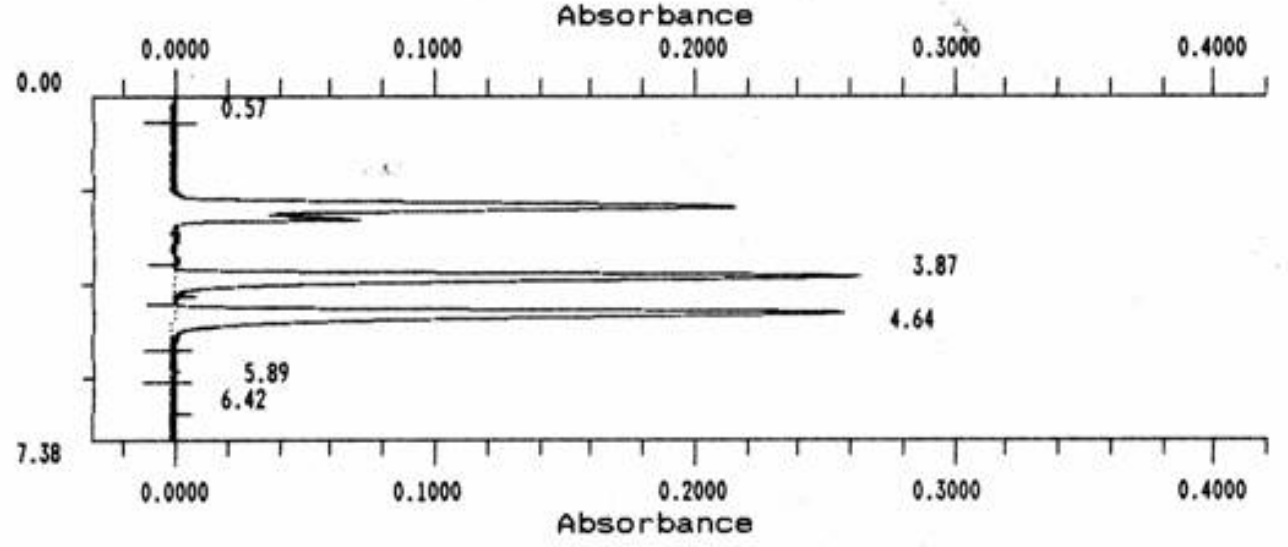

HPLC chromatogram for acetylated derivative of racemic 1-phenylpropy 1amine 
<smiles>CCC(NC(C)=O)c1ccccc1</smiles>

( - )

\begin{tabular}{ccccccccc} 
Type & $\begin{array}{c}\text { Saaple } \\
\text { Kaae }\end{array}$ & $\begin{array}{c}\text { Sanple } \\
\text { Anount }\end{array}$ & $\begin{array}{c}\text { Int Std } \\
\text { Anount }\end{array}$ & $\begin{array}{l}\text { Scale } \\
\text { factor }\end{array}$ & $\begin{array}{l}\text { Mr. } \\
\text { Inj }\end{array}$ & $\begin{array}{c}\text { Mial } \\
\text { Mr. }\end{array}$ & $\begin{array}{l}\text { Inject } \\
\text { Vol ut }\end{array}$ & \\
\hdashline Unk & 01173941 & & & 1.00000 & $1 / 1$ & & & (froe file)
\end{tabular}

\begin{tabular}{|c|c|c|c|c|c|c|c|c|c|c|c|}
\hline $\begin{array}{l}\text { Peak } R \\
\text { Mlaber }\end{array}$ & $\begin{array}{l}\text { Retention } \\
\text { Tinee }\end{array}$ & $\begin{array}{c}\text { Conponent } \\
\text { Nane }\end{array}$ & $\begin{array}{c}\text { Concentration } \\
\mathrm{g} / \mathrm{al}\end{array}$ & $\begin{array}{l}\text { Mornalized } \\
\text { Concentration }\end{array}$ & $\begin{array}{l}\text { Peak } \\
\text { Area }\end{array}$ & $\begin{array}{l}\text { Peak } \\
\text { Height }\end{array}$ & $\begin{array}{l}\text { Base } \\
\text { Code }\end{array}$ & $\begin{array}{l}\text { Response } \\
\text { factor }\end{array}$ & $\begin{array}{l}\text { Rel. Ret } \\
\text { Tine }\end{array}$ & $\begin{array}{l}\text { Area } \\
\text { Percent }\end{array}$ & $\begin{array}{l}\text { Height } \\
\text { Percent }\end{array}$ \\
\hline & & & & & :::: & :z:::z: & & & & & \\
\hline 1 & 0.230 & & 0.0000 & 0.000 & 0.03568 & 0.00053 & $\mathrm{BCV}$ & 0.0000 & 0.0000 & 0.035 & 0.1 \\
\hline 2 & 0.343 & & 0.0000 & 0.000 & 0.04748 & 0.00049 & VC8 & 0.0000 & 0.0000 & 0.046 & 0.100 \\
\hline 3 & 3.938 & & 0.0000 & 0.000 & 10.03512 & 0.05131 & $B C B$ & 0.0000 & 0.0000 & 9.720 & 10.386 \\
\hline 4 & 4.672 & & 0.0000 & 0.000 & 92.56631 & 0.43991 & BCB & 0.0000 & 0.0000 & 89.664 & 89.044 \\
\hline 5 & 6.149 & & 0.0000 & 0.000 & 0.55184 & 0.00179 & $B C B$ & 0.0000 & 0.0000 & 0.535 & 0.362 \\
\hline & & & & & & & & ... & & :::::: & \\
\hline DTALS & & & 0.0000 & .000 & 103.23643 & 0.49403 & & & & 100.000 & 10 \\
\hline
\end{tabular}

Average Efficiency: 0

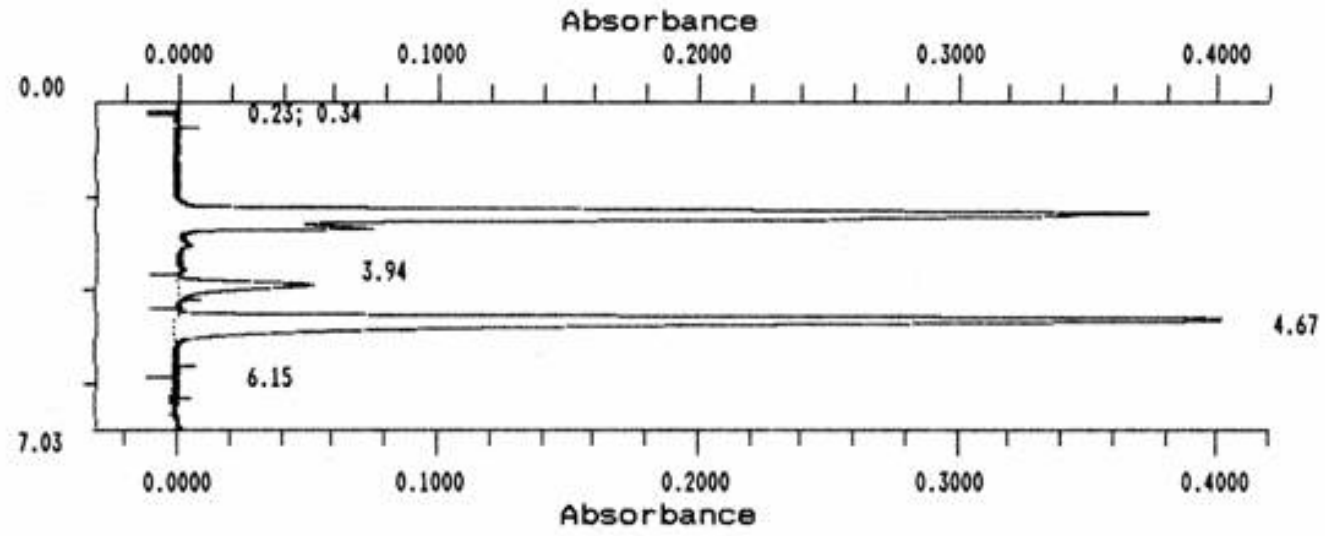

HPLC chromatogram for acetylated derivative of $(S)$-1-phenylpropylamine obtained from the reduction of O- $n$-butyl propionylphenone oxime 
<smiles>CCC(NC(C)=O)c1ccccc1</smiles>

$(-)$

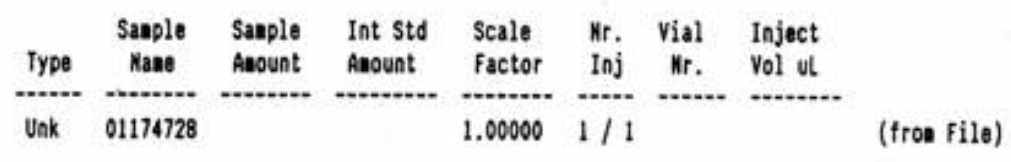

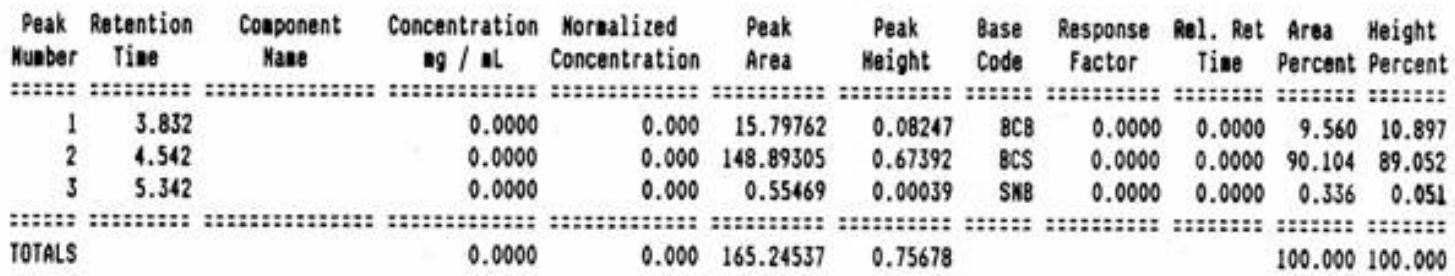

Average Efficiency: 0

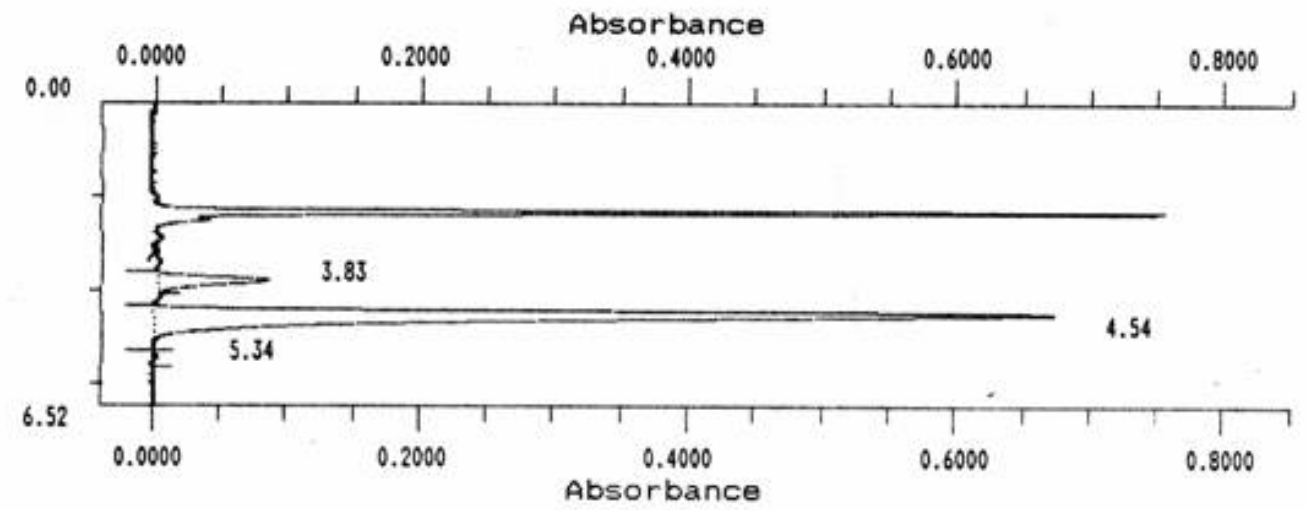

HPLC chromatogram for acetylated derivative of $(S)$-1-phenylpropylamine obtained from the reduction of O-benzyl propionylphenone oxime 
<smiles>CCC(NC(C)=O)c1ccc([N+](=O)[O-])cc1</smiles>

(土)

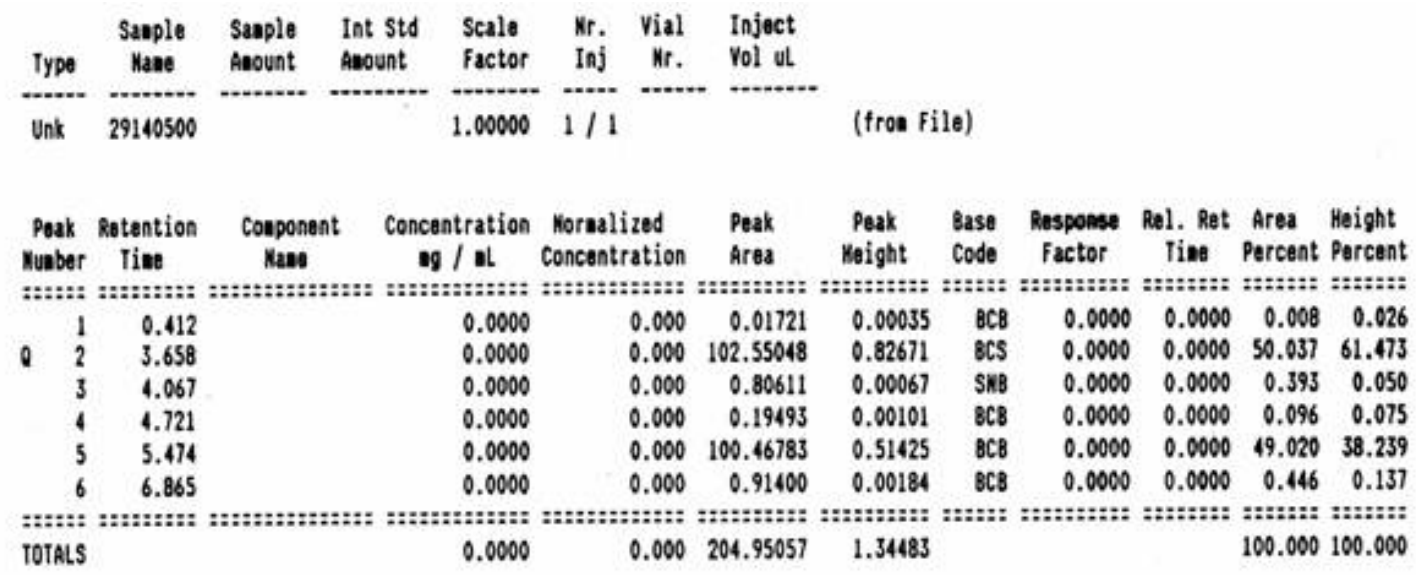

Average Efficiency: 0

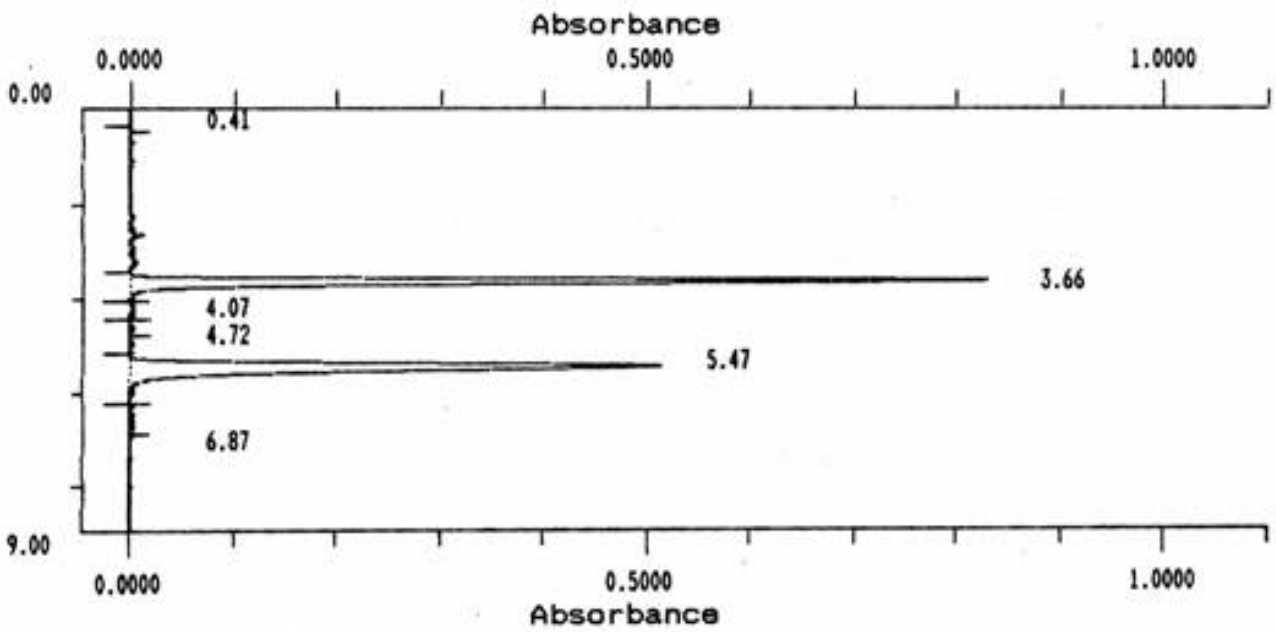

HPLC chromatogram for acetylated derivative of racemic 1-(4-nitrophenyl)ethylamine 
<smiles>CC(=O)NC(C)c1ccc([N+](=O)[O-])cc1</smiles>

$(-)$

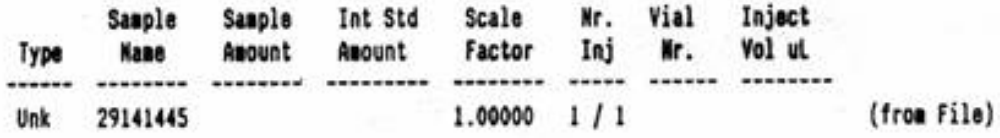

\begin{tabular}{|c|c|c|c|c|c|c|c|c|c|c|c|}
\hline $\begin{array}{l}\text { Peak } \\
\text { Nunber }\end{array}$ & $\begin{array}{l}\text { Retention } \\
\text { Tine }\end{array}$ & $\begin{array}{c}\text { Conponent } \\
\text { Mane }\end{array}$ & $\begin{array}{c}\text { Concentration } \\
\mathrm{g} / \mathrm{aL}\end{array}$ & $\begin{array}{l}\text { Moraslized } \\
\text { Concentration }\end{array}$ & $\begin{array}{l}\text { Peak } \\
\text { Area }\end{array}$ & $\begin{array}{c}\text { Peak } \\
\text { Meight }\end{array}$ & $\begin{array}{l}\text { Base } \\
\text { Code }\end{array}$ & $\begin{array}{l}\text { Response } \\
\text { factor }\end{array}$ & $\begin{array}{l}\text { Rel. Ret } \\
\text { Tíee }\end{array}$ & $\begin{array}{l}\text { Area } \\
\text { Percent }\end{array}$ & $\begin{array}{l}\text { Height } \\
\text { Percent }\end{array}$ \\
\hline & :z:z:Z: & & :::::: & & & ::::::::: & $::::$ & :::::::: & atmet:z & ::::::: & $:$ \\
\hline 1 & 0.335 & & 0.0000 & 0.000 & 0.00239 & 0.00008 & $B C B$ & 0.0000 & 0.0000 & 0.006 & 0.038 \\
\hline 2 & 3.492 & & 0.0000 & 0.000 & 0.80280 & 0. & $B C B$ & 00 & 000 & 1.893 & .530 \\
\hline 3 & 5.255 & & 0.0000 & 0.000 & 40.72330 & 0.20929 & BCS & 0.0000 & 0.0000 & 96.028 & 97.300 \\
\hline 4 & 5.925 & & 0.0000 & 0.000 & 0.87901 & 0.00028 & SMB & 0.0000 & 0.0000 & 2.073 & 0.132 \\
\hline & & & & & ::; & & & & & ::a:s: & \\
\hline & & & 0.0000 & 0.000 & 42.40750 & 0.21510 & & & & 100.000 & 100.000 \\
\hline
\end{tabular}

Average Efficiency: 0

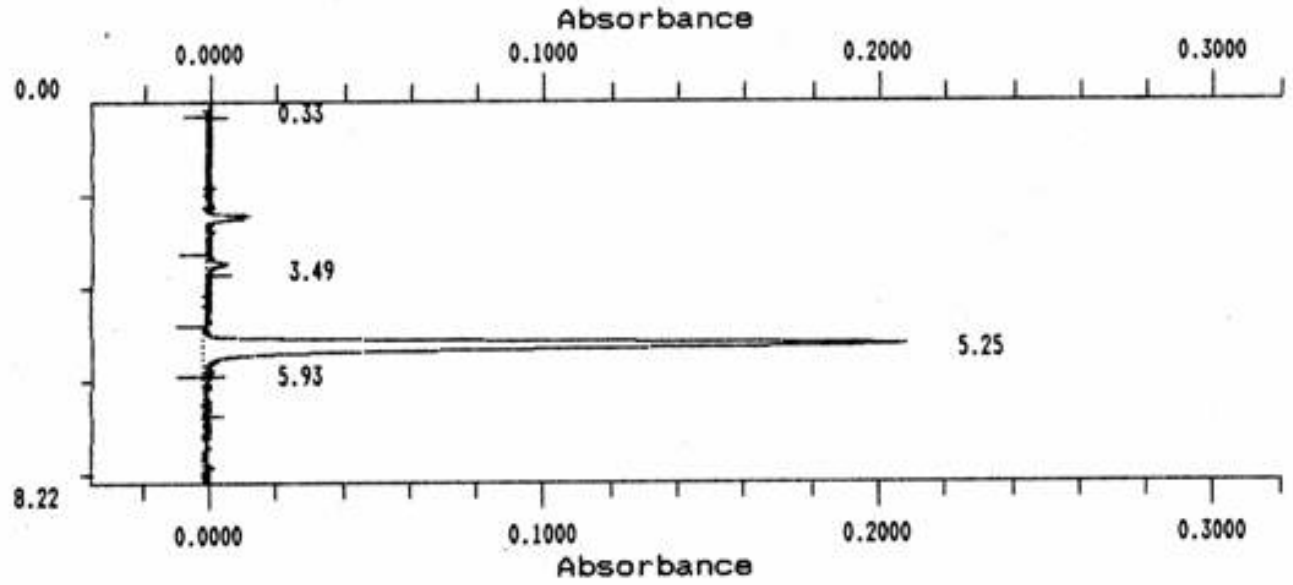

HPLC chromatogram for acetylated derivative of (S)-1-(4-nitrophenyl)ethylamine obtained from the reduction of O-methyl 4-nitrophenyl methyl ketoxime 
<smiles>CC(=O)NC(C)c1ccc([N+](=O)[O-])cc1</smiles>

$(-)$

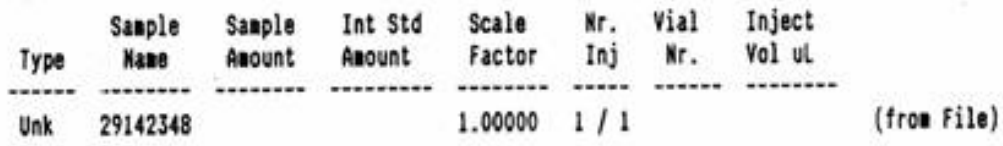

\begin{tabular}{|c|c|c|c|c|c|c|c|c|c|c|c|}
\hline $\begin{array}{l}\text { Peak } \\
\text { Nunber }\end{array}$ & $\begin{array}{l}\text { Retention } \\
\text { Tine }\end{array}$ & $\begin{array}{c}\text { Conponent } \\
\text { Nane }\end{array}$ & $\begin{array}{c}\text { Concentration } \\
0 / \mathrm{al}\end{array}$ & $\begin{array}{l}\text { Mornalized } \\
\text { Concentration }\end{array}$ & $\begin{array}{l}\text { Peak } \\
\text { Area }\end{array}$ & $\begin{array}{l}\text { Peak } \\
\text { Height }\end{array}$ & $\begin{array}{l}\text { Base } \\
\text { code }\end{array}$ & $\begin{array}{l}\text { Response } \\
\text { factor }\end{array}$ & $\begin{array}{l}\text { Rel. Ret } \\
\text { Tine }\end{array}$ & $\begin{array}{l}\text { Area } \\
\text { Percent: }\end{array}$ & $\begin{array}{l}\text { Height } \\
\text { Percent }\end{array}$ \\
\hline & & & : & . & :: & מ:m:s: & $::::$ & :::::::t: & 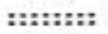 & & \\
\hline 1 & 0.244 & & 0.0000 & 0.000 & 0.04144 & 0.00056 & $B C B$ & 0.0000 & 0.0000 & 0.015 & 0.041 \\
\hline 2 & $x$ & & 0.0000 & 0.000 & 14.45663 & 0.11348 & 68 & 0.0 & 0.0000 & 171 & \\
\hline 3 & 5.276 & & 0.0000 & 0.000 & 264.63403 & 1.26002 & BCS & 00 & 00 & 94.666 & 91.691 \\
\hline 4 & 6.400 & & 0.0000 & 0.000 & 0.41335 & 0.00014 & SHB & 0.0000 & 0.0000 & 0.148 & 0.01 \\
\hline & & & & & & & & & & :::::: & \\
\hline & & & 0.0000 & 0.000 & 279.54546 & 1.37420 & & & & 100.000 & 100. \\
\hline
\end{tabular}

Average Efficiency: 0

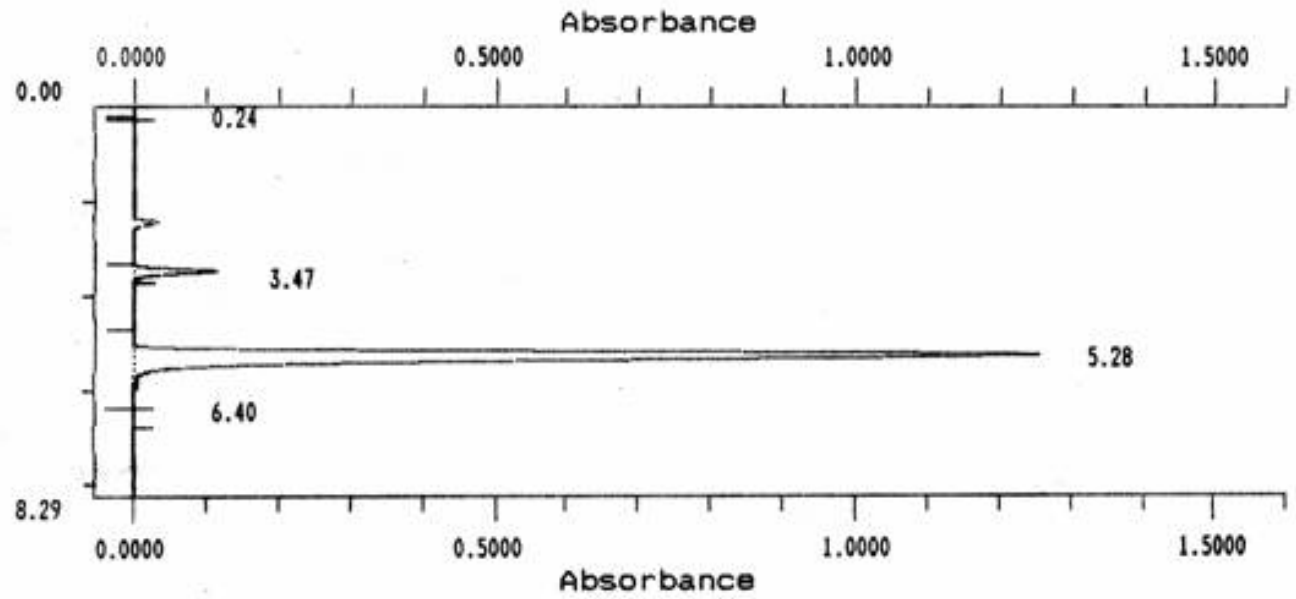

HPLC chromatogram for acetylated derivative of (S)-1-(4-nitrophenyl)ethylamine obtained from the reduction of O-benzyl 4-nitrophenyl methyl ketoxime 
<smiles>CC(=O)NC(C)c1ccc([N+](=O)[O-])cc1</smiles>

$(-)$

\begin{tabular}{|c|c|c|c|c|c|c|c|c|}
\hline Type & $\begin{array}{c}\text { Sanplo } \\
\text { Hase }\end{array}$ & $\begin{array}{l}\text { Saaplo } \\
\text { Amount }\end{array}$ & $\begin{array}{l}\text { Int Std } \\
\text { Aaount }\end{array}$ & $\begin{array}{l}\text { Scale } \\
\text { factor }\end{array}$ & $\begin{array}{l}\text { Mr. } \\
\text { Inj }\end{array}$ & $\begin{array}{l}\text { Vial } \\
\text { Mr. }\end{array}$ & $\begin{array}{l}\text { Inject } \\
\text { Vol ut }\end{array}$ & \\
\hline & 30093753 & & & 1.00000 & $1 / 1$ & & & (frou File) \\
\hline
\end{tabular}

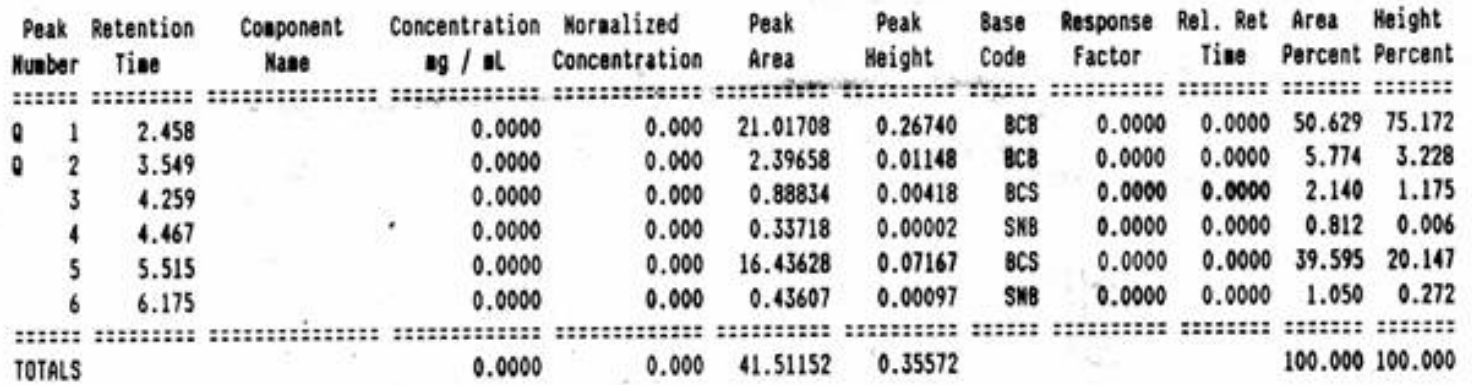

Average Efficiency: 0

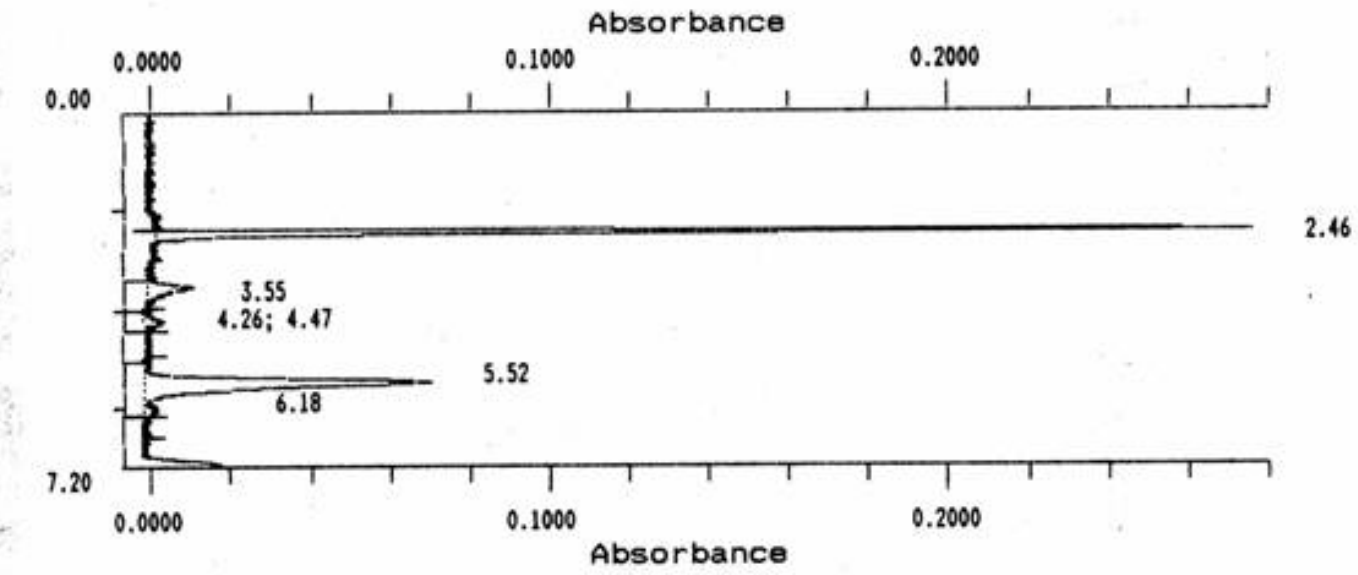

HPLC chromatogram for acetylated derivative of (S)-1-(4-nitrophenyl)ethylamine obtained from the reduction of O-n-butyl 4-nitrophenyl methyl ketoxime 